\title{
Assessing Integrative Complexity as a predictor of morphological learning using neural networks and artificial language learning
}

\author{
Tamar Johnson, Jennifer Culbertson, Hugh Rabagliati and Kenny Smith \\ Centre for Language Evolution, University of Edinburgh, Department of Psychology, University of \\ Edinburgh
}

\begin{abstract}
Morphological paradigms differ widely across languages: some feature relatively few contrasts, and others, dozens. Under the view that languages are under pressure to be learnable and that the distribution of languages in the world reflects biases in language learning, this diversity is surprising - how could paradigms which apparently differ so markedly be similarly learnable? Recent work on morphological complexity has argued that even very large paradigms are designed such that they are easy to learn and use. Specifically, Ackerman \& Malouf, 2013 propose an information-theoretic measure, i-complexity, which captures the extent to which forms in one part of a paradigm predict forms elsewhere in the paradigm, and contrast this measure with e-complexity, which captures the number of distinctions made by the language and the different ways to mark each grammatical function. They show that languages which differ widely in e-complexity exhibit similar i-complexity; in other words, morphological paradigms with many contrasts reduce the learnability challenge for learners by having predictive relationships between forms. Here, we test whether i-complexity predicts the learnability of inflectional paradigms using both recurrent neural networks and human participants trained on an artificial language. Furthermore, we compare the effect of i-complexity on learning with that of e-complexity. We find that in RNNs both i- and ecomplexity have an effect on learning: paradigms with lower i- and e-complexity are easier to learn, although the effect of e-complexity is larger. However, for human learners, we find only weak evidence (if any) that low i-complexity paradigms are easier to learn; in contrast, low e-complexity is clearly beneficial for learning. This suggests that i-complexity might have relatively little influence on the learnability of inflectional paradigms, with other factors, such as the e-complexity having a greater effect. These results suggest that appealing to i-complexity does not fully resolve the paradox of cross-linguistic variation in morphological systems.
\end{abstract}

Keywords: morphological complexity; inflection paradigm; neural network; LSTM; artificial language learning; entropy

\section{Introduction}

There is substantial variation in inflectional paradigms cross-linguistically. Some languages are largely devoid of inflectional morphology (e.g., Vietnamese), while others have rich inflectional systems, marking dozens of grammatical functions (e.g., Arabic). Determining the dimensions of variation in inflectional systems has been an important goal for linguistics (e.g., Sapir, 1921; Bybee, 1995; Bickel \& Nichols, 2007). However from the perspective of language learning, the existence of highly complex inflectional paradigms poses a challenge. In particular, it is often argued that languages are shaped by an inductive bias for simplicity (Chater \& Vitanyi, 2003; Pothos \& Chater, 2003; Chater, Clark, Goldsmith, \& Perfors, 2015; Kirby, Tamariz, Cornish \& Smith 2015; Feldman, 2016; Culbertson \& Kirby, 2016). According to this view, simplicity is a general principal guiding learning across cognitive domains: learners are biased towards inferring simpler hypotheses to explain observed data. In the linguistic domain, this principal has been described as a preference for languages whose grammars can be expressed more compactly (Culbertson \& Kirby, 2016). Effects of this simplicity bias on learning have been consistently reported in laboratory experiments in which learners are trained on miniature artificial languages (e.g., Saffran \& Thiessen 2003; Culbertson, Smolensky \& Legendre 2012; Kirby, Tamariz, Cornish \& Smith 2015; Canini, Griffiths, Vanpaemel, and Kalish, 2014, a.o.). These experiments show that individual learners are less able to learn more complex linguistic patterns, infer simpler patterns whenever possible, and drive languages to become simpler over simulated generations of learners. On learnability grounds, the expectation is therefore that simple morphological paradigms should dominate cross-linguistically. ${ }^{1}$ At

\footnotetext{
${ }^{1}$ Of course, learnability is not the only factor shaping linguistic systems: languages are used for communication, and linguistic systems have been claimed to reflect a trade-off between the bias for simplicity (i.e., minimizing the system's complexity) and pressure from communication favoring languages with higher expressivity (i.e., minimizing ambiguity, Kemp \& Regier 2012). This trade-off has been shown in a variety of linguistic domains, where natural languages show a near optimal balance between these two pressures (e.g., Regier et al. 2015; Y. Xu, Regier, \& Malt 2016; Zaslavsky et al. 2019). Evidence for this trade-off has also been found in experimental studies manipulating the relative importance of learning and communication (e.g. Silvey, Kirby \& Smith 2015; Kirby, Tamariz, Cornish and Smith
} 
first glance, this appears to be contradicted by the existence of many languages with highly complex paradigms, which have been maintained over many generations. However, in recent work, Ackerman and Malouf (2013) argue that apparently complex morphological paradigms are in fact relatively simple when complexity is measured not in terms of number of forms, but how predictable forms are from each other.

\section{I-complexity vs. e-complexity}

Ackerman and Malouf (2013) discuss two measures of complexity: enumerative complexity (ecomplexity) and integrative complexity ( $i$-complexity). The e-complexity of a language reflects the number of grammatical functions and morphosyntactic categories words in the language are marked for, the number of different forms to mark each category and their type frequencies within the morphological paradigm (Meinhardt, Ackerman \& Malouf, 2019). This measure of complexity is seen to express complexity according to the item and arrangement theories of morphology (Hockett, 1954) that take the morpheme as the fundamental unit of analysis. Therefore, the learner's task when learning the morphological system of a language is to create an inventory of the affixes and their meanings. Icomplexity is motivated by the idea that paradigms in which new forms can be easily predicted by old forms are simpler. This measure fits naturally within the word and paradigm theories of morphology in which the relationship among forms in a paradigm, and not just the forms themselves, is a crucial feature of how paradigms are represented and processed (e.g., Stump, 2001; Finkel \& Stump, 2007; Blevins, 2006; Blevins et al., 2016). Intuitively, since exposure to lexical items is relatively sparse (i.e. a learner is unlikely to have experienced all forms of a lexeme before producing them), learners must use the forms they have heard to predict unknown forms (the Paradigm Cell Filling Problem, Ackerman \& Malouf, 2009). Ackerman and Malouf (2013) suggest an information-theoretic measure for I-complexity derived from Shannon entropy (Shannon, 1946). It quantifies how difficult this prediction will be, namely how well one inflectional form can predict the other. This is calculated using the conditional entropy (or uncertainty) of one inflectional form Y given another X in the paradigm, as in (1) below:

$H(Y \mid X)=H(X, Y)-H(X)=\sum_{x \in X} \sum_{y \in Y} P(x, y) \log _{2} P(y \mid x)$

Take for example the nominal plural paradigm in Modern Greek (Table 1), which expresses four cases, with a series of different morphemes that depend on the inflectional class of the word. If a word takes $-i$ in the nominative plural, it must be in inflectional class 1 or 7 . Knowing this reduces the uncertainty of the accusative form: it must be $-u s$ or $-i$ (not $-e s$, $-i s$ or $-a$ ). By contrast, knowing that a word takes - on in the genitive plural does not provide any information about inflectional class, because the genitive is marked with -on across classes, and so it does not reduce uncertainty about the accusative form. Therefore, in Greek, $\mathrm{H}($ acc.pl|nom.pl) is lower than $\mathrm{H}(\mathrm{acc} . \mathrm{pl} \mid$ gen.pl). To calculate the overall i-complexity of a paradigm, one simply averages conditional entropy over all pairs of inflections.

\begin{tabular}{lllll}
\hline & \multicolumn{4}{c}{ Plural } \\
Class & Nom & Gen & Acc & Voc \\
\hline 1 & $-i$ & $-o n$ & $-u s$ & $-i$ \\
2 & $-e s$ & $-o n$ & $-e s$ & $-e s$ \\
3 & $-e s$ & $-o n$ & $-e s$ & $-e s$ \\
4 & $-i s$ & $-o n$ & $-i s$ & $-i s$
\end{tabular}

2015; Motamedi et al. 2019). Morphological paradigms which appear highly complex could in principle reflect a balance between the communicative needs of speakers and the inductive biases of learners. However, the existence of inflectional classes-groups of lexemes that share the same set of inflectional realizations (Aronoff, 1994; Corbett, 2009)-which add to the complexity of paradigms without any countervailing benefit (Baerman et al., 2010) make an analysis along these lines non-straightforward at best. 


\begin{tabular}{lllll}
5 & $-a$ & - on & $-a$ & $-a$ \\
6 & $-a$ & - on & $-a$ & $-a$ \\
7 & $-i$ & - on & $-i$ & $-i$ \\
8 & $-a$ & - on & $-a$ & $-a$ \\
\hline
\end{tabular}

Table 1. Modern Greek plural nominal inflectional classes (Ackerman \& Malouf, 2013 based on Ralli, 2002). Columns give the inflectional endings for nouns in different grammatical roles (nominative, genitive, accusative and vocative), for plural; rows show the 8 inflectional classes in Modern Greek.

Information theory can also be used to characterise e-complexity. Specifically, Ackerman and Malouf (2013) propose an entropy based measure of e-complexity, that captures the difficulty in choosing the correct inflection for a lexeme based only on the set of possible markers for that inflection in the language. Being based on entropy, this measure is more subtle than merely counting inflection classes or variants of realization in the paradigm. We therefore adopt this measure as the e-complexity of a morphological paradigm.

E-complexity will be higher for paradigms with more inflectional features marked, and more different ways to mark them. It is calculated as in (2) below, again summing over all inflections $\mathrm{X}$ in a paradigm.

$H(X)=-\sum_{x \in X} p(x) \log _{2} p(x)$

This measure captures the intuition that the Greek nominal inflection paradigm is complex because it makes a large number of distinctions and uses a variety of endings to do so. In the case of plural nouns in Modern Greek, e-complexity captures something very different from i-complexity. While the nominative forms contribute less i-complexity to the paradigm than genitives (because they are informative about other cells in the paradigm), nominative forms have higher e-complexity than genitive forms (because there are several different nominative markers but only one genitive; in fact $\mathrm{H}($ gen.pl $)=0$ ).

\section{Evidence for i-complexity as a predictor of learnability}

Ackerman and Malouf (2013) argue that i-complexity rather than e-complexity captures what is difficult about learning and using an inflectional paradigm, and that this explains why languages show such diversity in apparent morphological complexity: Apparently complex languages have a simple integrative structure. This argument generates two clear predictions. First, if two inflectional paradigms differ only in i-complexity, the paradigm with lower i-complexity should be learned more easily. Second, if paradigms with lower i-complexity are simpler to learn, they should be more common cross-linguistically (i.e., cultural transmission should lead to decreases in i-complexity which compound over generations, Kirby et al., 2015). Ackerman and Malouf (2013) provide some evidence for the second prediction: they show that, across a set of 10 geographically and genetically different languages, e-complexity varies quite widely (from 0.78 to 4.9 bits) but i-complexity is consistently low (around 0.6 bits for all languages in their sample). Moreover, they demonstrate, using a Monte-Carlo simulation on one of the languages in their sample (Chiquihuitlàn Mazatec), that the i-complexity of the verbal inflectional system of the language is minimized compared to all other permutations of the markers in the paradigm; in other words, the configuration of the paradigm is such that $\mathrm{i}$-complexity is lower than we would expect if the paradigm forms were organized randomly. Both of these pieces of evidence suggest that paradigms are implicitly designed to minimise i-complexity. ${ }^{2}$

\footnotetext{
${ }^{2}$ It is worth noting that these findings have been criticized on the basis that calculating the i-complexity of languages' paradigms is highly dependent on linguists' analysis of the language and their decisions on how to describe its paradigms (e.g., Bonamie \& Beniamine, 2016; Sims \& Parker, 2016).
} 
However, there is only limited evidence in support of the first prediction, namely, that morphological paradigms with lower i-complexity are easier to learn. Recall that i-complexity represents the extent to which one inflectional form in a paradigm can predict another. For example, if a learner can infer the inflectional class membership of a word from its realization in one inflectional category, then new inflectional forms of the word can be predicted even if they have not been previously encountered. One way for this to happen is through analogy; if a word behaves like another in one inflectional category, then by analogy it will behave like that word in another inflectional category (Stump, 2001; Blevins, 2006; Blevins et al., 2016; Ackerman \& Malouf, 2013; Malouf, 2017). Setting aside for a moment the role of icomplexity, we might ask first whether there is evidence that learners will take that analogical step. A number of previous studies suggest that adults and children will choose inflections for novel words based on their phonological similarity to familiar words (e.g., Milin, Keuleers \& Đurdevic', 2011; Ambridge, 2010). In addition, there is evidence from artificial language learning experiments that learners' willingness to assume that a word belongs to a particular category is influenced by perceived level of similarity among forms (e.g., Frigo \& McDonald, 1998; Gerken et al., 2009; Reeder et al. 2013; Culbertson et al. 2017). These results are all compatible with the idea that learners build relations between forms in part by analogy. However, they do not test the idea that predictive relationship among forms in the paradigm are critical for learning. Further, they suggest that analogy may be dependent on features of the word stems (i.e. their phonological or semantic similarity), not relations among the inflections themselves. Although Frigo \& McDonald (1998) show evidence for the ability of learners to use distributional cues for novel nouns when phonological similarities are provided.

Seyfarth, Ackerman and Malouf (2014) directly test the effect of i-complexity on completing the Paradigm Cell Filling Problem - in which learners must learn a subset of forms in a paradigm, and then use those forms to predict new ones. In their Experiment 1, participants were exposed to a miniature artificial language with word forms from the nominal paradigm shown in Table 2A, with two inflectional classes, and three numbers. Class membership was not cued by anything other than the pattern of inflectional endings. The paradigm's key feature is that the inflection in the dual is predicted by the word form in the singular (and vice versa), whereas the form of the plural is not predictive of any other inflectional form (since it has the same form for both inflectional classes). After learning the paradigm, participants were asked to choose the correct forms for novel stems, either in predictive trials (i.e., providing the word form in singular having been given the dual form) or in non-predictive trials (generating the singular or dual given the plural). They found that participants were more likely to generate the correct form on predictive trials than non-predictive trials. In their Experiment 2, they tested whether predictive information facilitates generalization to novel stems in a larger paradigm (Table 2B). Results from this experiment suggested that learners tended to inflect novel stems with the most frequent marker (e.g., they used the suffix -cav to mark dual regardless of class). However they did use predictive information to generalize to novel stems when the predicted suffix was low-frequency.

\begin{tabular}{lccc}
\hline & Singular & Dual & Plural \\
\hline noun class 1 & -yez & -cav & -lem \\
noun class 2 & -taf & -guk & -lem \\
\hline
\end{tabular}

A

\begin{tabular}{lccc}
\hline & Singular & Dual & Plural \\
\hline noun class 1 & -taf & -guk & -lem \\
noun class 2 & -yez & -cav & -lem \\
noun class 3 & -yez & -cav & -nup \\
\hline
\end{tabular}
B

Table 2. Example nominal inflection paradigm used in Seyfarth et al., (2014). A paradigm with two noun classes as in Experiment 1 (A), and one of the paradigms used in Experiment 2 with three noun classes (B).

Further evidence comes from Malouf (2017), who trained recurrent neural networks on natural language corpora in order to test whether they could predict unobserved forms after learning partial paradigms. The 
networks were trained to predict phonological forms based only on a lexeme (an abstract representation of related forms without phonological information) and a set of inflectional features (e.g. tense or number). Networks were then tested on their accuracy at producing phonological forms for lexemes with untrained inflectional features. Across a number of languages, these networks outperformed baselines which learned based on phonological information (lemmas rather than abstract lexemes) combined with specialized rules for guessing affixes. Together, these findings suggest that learners can generalize to novel inflection forms based on analogy with known forms, and further that this is facilitated by predictive links between forms in a paradigm ${ }^{3}$.

However, the task used in both cases serves as a relatively low bar for testing the facilitative effect of low i-complexity. For example, Seyfarth et al. (2014) compare predictive cases, where participants can generalize by analogy with learned forms, with cases where participants have no basis for generalizing (and must therefore simply guess). Therefore, it is in a sense not surprising that participants are better at determining the correct form in the former than the latter. The results of their second experiment in fact suggest that when learners have access to other cues for generalizing, e.g. marker frequency, it is less clear that learners use the kind of predictive information captured by i-complexity. Notably, marker frequency is captured by e-complexity. More generally, both Seyfarth et al. (2014) and Malouf (2017) simulate cases in which language learners have to generalize from the partial paradigm they have learned to express an entirely new form they have never been exposed to. This is an extreme form of a much more general problem that language learners face; in some cases, learners must generalize to entirely novel forms, in many others, they must generate (or retrieve) forms which may have been encountered but have not yet been robustly acquired. In principle, prediction should facilitate learning in all these cases.

\section{The present study}

Here we build on Seyfarth, Ackerman and Malouf (2014) by testing whether i-complexity affects how humans and neural networks learn morphological paradigms. We use artificially constructed paradigms which vary in i-complexity controlling for other factors, such as e-complexity, number of markers, and phonological similarity among stems. In a series of simulation experiments we test whether lower values of i-complexity can facilitate the learning of inflectional paradigms for recurrent neural networks (RNNs): we train Long Short Term Memory (LSTM) RNNs on two inflectional paradigms, differing only in their i-complexity values, and show that RNNs generalize more successfully to new forms in low i-complexity paradigms and that paradigms with lower i-complexity are learnt faster. We then test whether low icomplexity also facilitates learning for human learners: we use the same artificial languages used for the RNNs, and train human participants on the same two inflectional paradigms, differing only in their icomplexity. In addition, we compare the effect of i-complexity on learning with that of e-complexity by training RNNs and human participants on an inflectional paradigm with high e-complexity. These experiments reveal only weak evidence of an advantage for low i-complexity languages in paradigm learning; in contrast, low e-complexity is clearly beneficial for learning. These findings suggest the

\footnotetext{
${ }^{3}$ Marzi et al. (2018) give support to Ackerman and Malouf's conjecture from a different angle, providing evidence to their hypothesis that the e-complexity of the morphological system is not the main factor affecting its learnability. They propose a model for simulating learning morphological systems using a recurrent neural network trained on inflected forms and show that changes in the e-complexity of the input language does not strongly affect the learning of the morphological paradigm by the model. They trained the neural network on six languages with varying levels of e-complexity (Greek, Italian, Spanish, German, English and Arabic) and compared how successful the networks were at predicting the inflected forms across these languages. They evaluated how accurately the networks could predict incrementally presented words and found little variance in the model's results for the different languages, with a significant difference in the prediction accuracies of the model only between the most e-complex language (Modern Greek) and the least e-complex language (English). However, Marzi et al. (2018) use word prediction to test the model's learning of the forms in the paradigm without any morphological information on the inflected form given as input. Rather, the model predicts each consecutive character in the form solely based on the string of characters already presented. Therefore, it is not clear whether the results from this study provide clear evidence for Ackerman and Malouf's hypothesis, or whether these results are instead due to the use of a task which does not include a key type of information used by learners.
} 
possibility that although i-complexity may affect learning, it is not the central measure of morphological complexity as experienced by the language learner.

\section{Testing the impact of i-complexity on paradigm learning in Recurrent Neural Networks}

Neural network models of learning are inspired loosely by the structure of networks of neurons in the brain. Artificial neural networks consist of interconnected nodes, each with an activation value. Activation spreads from node to node via weighted connections - activation at one node will spread to other nodes with which it has positively-weighted connections, increasing their activation, and will drive down the activation of nodes with which it has negatively-weighted connections. In the types of networks we use here, nodes are arranged in layers. Nodes within one layer are connected via unidirectional weights to nodes in a subsequent layer. The first layer of nodes in the network is called the input layer and the last one is the output layer. The middle layer(s) are called hidden layers. The network weights are learned via supervised training on pairs of data points, each pair consisting of an input pattern of activation with a desired output pattern of activation. For example, the input might be the singular form of a word, and the output the corresponding plural. During training, the neural network connections are tuned with the objective of approximating the function from input patterns presented at the input layer to output patterns in the output layer. Specifically, connection weights are updated through a process called backpropagation which optimizes the weights so that when the network encounters some input pattern of activation, it produces the desired pattern of activation over the output layer. After training, the weights are fixed and the network can be tested with completely new data points or with data points similar to those presented during training (depending on the task).

Recurrent neural networks add 'short term memory' to the network, by looping back the output or hidden layer activations previously produced for earlier inputs (Elman, 1991; Elman, 1990; Jordan, 1986). This allows networks to make predictions based on sequences of inputs; for example, when predicting the next word in a sentence, the ability to keep previous words in memory is critical. In RNNs, the extent to which previous inputs affect the processing of the current input is also determined by weights, optimized through backpropagation.

Long Short Term Memory (LSTM) networks are an extension of recurrent neural networks introduced by Hochreiter and Schmidhuber (1997) in order to improve learning of longer temporal dependencies. Practically, LSTMs add an element of 'long term memory' to networks by allowing the network to control the influence of current and previous inputs during the process of activation propagation, using 'gates' in the networks. Like activation weights, network gates are optimized during training to determine what information is stored or passed along and therefore allowed to influence subsequent inputs. This allows LSTMs to make better use of sequential information, including learning sequential dependencies with long time lags.

A series of recent studies testing LSTM network on language tasks provide evidence that LSTM networks are capable of learning complex linguistic structure, and in some cases performance is similar to that of human participants. For example, Linzen, Dupoux and Goldberg (2016) show that LSTM networks can predict long-distance subject-verb number agreement, even in the presence of other potential agreement triggers (often called attractors) intervening between the subject and verb. Gulordava et al. (2018) show that LSTMs trained on four different languages can accurately predict subject-verb agreement even when they are not trained specifically on that task. Futrell and Levy (2018) show that surprisal scores of LSTMs (a measure of successful prediction) mirrored preferences of human participants on grammatical judgments task differentiating word-order alternations.

LSTMs therefore offer a powerful but convenient general-purpose learning mechanism for modelling human learning. Here we use LSTMs to process relatively short sequences: we train models on artificially constructed inflectional paradigms which differ only in their i-complexity. The networks are trained and tested on wordforms plus suffixes. We then test them on (i) whether they are able to exploit 
the predictive information present in the lower i-complexity paradigm in order to generalise to novel forms, and (ii) whether the inflectional paradigm with lower i-complexity is learnt faster.

\section{Method}

\section{Target Paradigms}

We constructed two paradigms, which we used to test the effect of i-complexity in neural network and human learners. The basic paradigms both consisted of nine CVC nouns (gob, tug, sov, kut, pid, tal, dar, ler, mip), randomly paired with meanings for human participants (see Testing the impact of i-complexity on paradigm learning in humans below). An additional nine nouns were used to test network generalization (bor, ges, kiv, mas, nek, nap, lan, wib, log) in Simulation Experiment 1 below. The small lexicon size allows the system to be learned with reasonable accuracy by human participants in a short experiment. The nouns were randomly allocated to three classes (for each run of the network, or each human participant), and each class was inflected for three numbers: singular, dual and plural. As in Seyfarth et al. (2014) noun class membership was not indicated by the semantics or phonology of the noun stem, but was rather defined only by different patterns of inflection. Inflectional markers were seven VC monosyllabic suffixes (-op, $-o c,-u m,-i b,-e l,-o d,-a t)$. These inflectional markers were randomly allocated to cells in each paradigm (for each run of the network, or each human participant) such that both paradigms shared the same e-complexity value (1.14 bits) but differed in i-complexity. In the low i-complexity paradigm, the singular form of a word predicts the dual form, while in the high i-complexity paradigm it does not. Tables 4 shows two example paradigms. In the low i-complexity paradigm (A), if a stem takes the marker - at in singular, then it takes -oc in dual; if a stem takes -op in singular, then it takes -um in the dual. In contrast, in the higher i-complexity paradigm (B), there is not such regularity: nouns with -at in the singular take either $-o c$ or $-u m$ in the dual. The i-complexity value for the low i-complexity language is 0.222 bits vs. 0.444 bits for the high i-complexity language. Note that the distinct plural forms in each paradigm serve to separate the three classes of nouns. Without distinct plural forms, the low i-complexity paradigm would have fewer classes and then high i-complexity paradigm.

\begin{tabular}{lccc}
\hline & Singular & Dual & Plural \\
\hline noun class 1 & - at & $-o c$ & $-\mathrm{ib}$ \\
noun class 2 & $-\mathrm{op}$ & $-\mathrm{um}$ & $-\mathrm{el}$ \\
noun class 3 & $-\mathrm{at}$ & $-\mathrm{oc}$ & $-\mathrm{od}$ \\
\hline
\end{tabular}

A

Table 4. Example paradigm for low i-complexity language (A) and high i-complexity (B).

Learning in both computational and behavioral studies was staged - neural networks and human participants were first trained on the forms of the stems in singular and plural before being exposed to the forms in dual. This should increase the likelihood of learners exploiting the predictive relationships in the paradigm. The critical measure of learning was accuracy on dual forms, although we also report accuracy for singular and plural forms.

\section{LSTM model}

We implemented LSTM networks using the Keras package in Python (Chollet, 2015). Figure 1 presents a diagram of the network, which consists of an input layer, a hidden layer of LSTM units, and an output layer fully connected to the hidden layer. In this task, the LSTM network takes as input a string (a sequence of characters) representing a noun stem and a number indicating the grammatical number of the object ( 1 for singular, 2 for dual and 3 for plural), e.g., the sequence sov3 indicates the stem sov in plural. The input string is fed into the network incrementally. The network has seven output units, one for each of the 
inflectional suffixes in the target paradigms. The model is trained to generate the correct suffix for a stem, number sequence. Both input stem+number sequences and output suffixes were encoded as one-hot vectors. i.e., every character (and number) used in the language is represented as a vector of zeroes (with length equal to the total set of characters, 27, for the input, and the total set of suffixes, 7, for the output) with ' 1 ' in a different index uniquely identifying it. The algorithm used Stochastic Gradient Descent (SGD) to update the weights of the network during training.

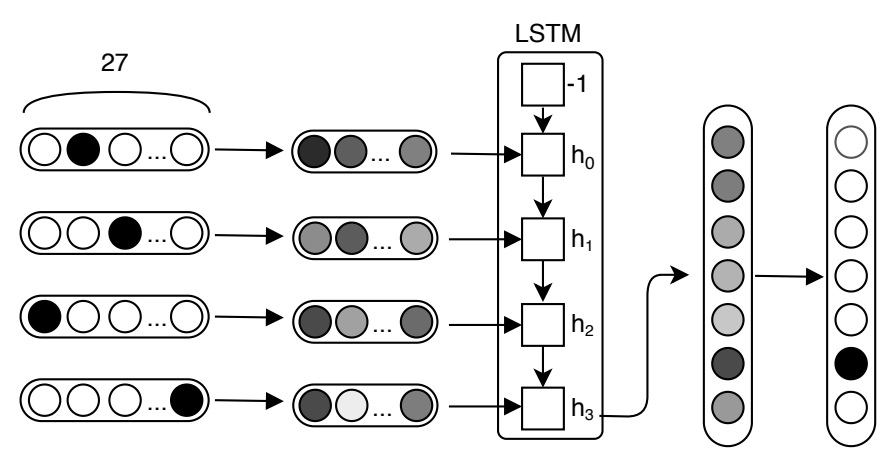

Figure 1. A diagram of the recurrent neural network: the input layer receives a sequence of four characters (stem + grammatical number), each coded as a one hot vector with length equal to the number of characters in the language (27). The input vectors are embedded, and embeddings are transferred to a hidden layer with LSTM units. Output from the LSTM units $\left(h_{3}\right)$ is fully connected to an output layer with seven options, representing the seven suffixes in the language. Using a SoftMax function, the output is converted back to a one hot vector, representing the suffix the network selected for this input.

\section{Simulation Experiment 1 - generalizing to novel forms}

We first tested whether low i-complexity facilitates solving the Cell Filling Problem (Ackerman \& Malouf, 2009) in RNNs. We trained RNNs on nine stems in the full paradigm, and tested their accuracy at producing the correct dual suffix for nine additional stems, for which the network was trained on the singular and plural form but never the dual (i.e. for these 9 stems the network was required to generalise to the dual based on its representation of the full paradigm and its exposure to the singular and dual forms for those 9 stems). Stems were assigned randomly to one of the three noun classes in the language, such that each noun class included six stems (three fully trained, and three with dual held out).

For each paradigm, the model was trained and tested on input-output pairs in three blocks, summarized in Table 5 below. In block 1, the network was trained and tested on singulars for all stems; in block 2 the networks was trained and tested on singulars and plurals for all stems; in block 3 the network was trained on singulars and plurals for all stems, plus duals for nine of the 18 stems. Finally, it was tested on the entire paradigm for all stems (i.e. including duals for the 9 stems where the dual form was held-out in training). Each block consisted of 300 epochs, each consisting of a single pass through the specified training set (randomized) with weights updated by backpropagation, followed by a pass through the specified test set (randomized). During testing, the network was given an input and had to generate an inflection. As noted above, weights in the network are tuned only during training, while during testing the network is tested on the same input without updating weights. Our results show performance in the testing phase.

\begin{tabular}{llll}
\hline Block & Epochs & Training & Testing \\
\hline 1 & 300 & all stems, singular only (18 trials) & all stems, singular only (18 trials)
\end{tabular}




\begin{tabular}{llll}
2 & 300 & all stems, singular and plural (36 trials) & all stems, singular and plural (36 trials) \\
3 & 300 & 9 fully-trained stems; 9 dual-held-out stems (45 trials) & all stems, all numbers (54 trials) \\
\hline
\end{tabular}

Table 5: Summary of training and testing blocks for RNNs in Simulation Experiment 1.

Since we did not have a hypothesis regarding the appropriate network size for this task, we varied the network size, from 5-cells networks (582 parameters) to 45 (12,382 parameters), in increments of 5. For each network size, we conducted 100 runs of the model for each paradigm. In each run of the model, the initial weights were randomly generated, according to a 'glorot_uniform' function (sampling from a uniform distribution in the range of $[-x,+x]$, where $\mathrm{x}$ is a function of the size of the network).

\section{Results}

Figure 2 presents the mean accuracy (averaged over all runs) on dual forms for dual-held-out stems by networks trained on the two paradigms. Networks trained on the low i-complexity paradigm achieved higher accuracy than networks trained on the high i-complexity paradigm across all sizes of the network; in other words, networks trained on the low i-complexity paradigm were more accurate in generalizing the paradigm to forms they were not trained on. Note that this is the case even though, in both the low icomplexity and high i-complexity paradigms, it is possible to infer the dual form of these nouns (untrained) from their plural forms (trained). For example, looking at the high i-complexity paradigm in Table 4B, if the plural form of the stem sov is sovib, then it must be in noun class 1 , and therefore its form in dual should be sovum. The predictive function of the plural thus allows networks trained on both paradigms to reach high accuracy, but there is nonetheless a clear advantage to the network trained on the low i-complexity language, where the singular provides an additional cue. In the Appendix we show results for networks trained only on the singular forms of the novel stems (rather than exposing them to both the singular and plural forms): under that training regime, the networks trained on the high icomplexity paradigm show accuracy of around $66 \%$ on generating duals for novel stems (chance level when guessing the more frequent dual suffix), while networks trained on the low i-complexity paradigm still show accuracy of almost $100 \%$ when generalizing to the dual (see Appendix Figure 14).
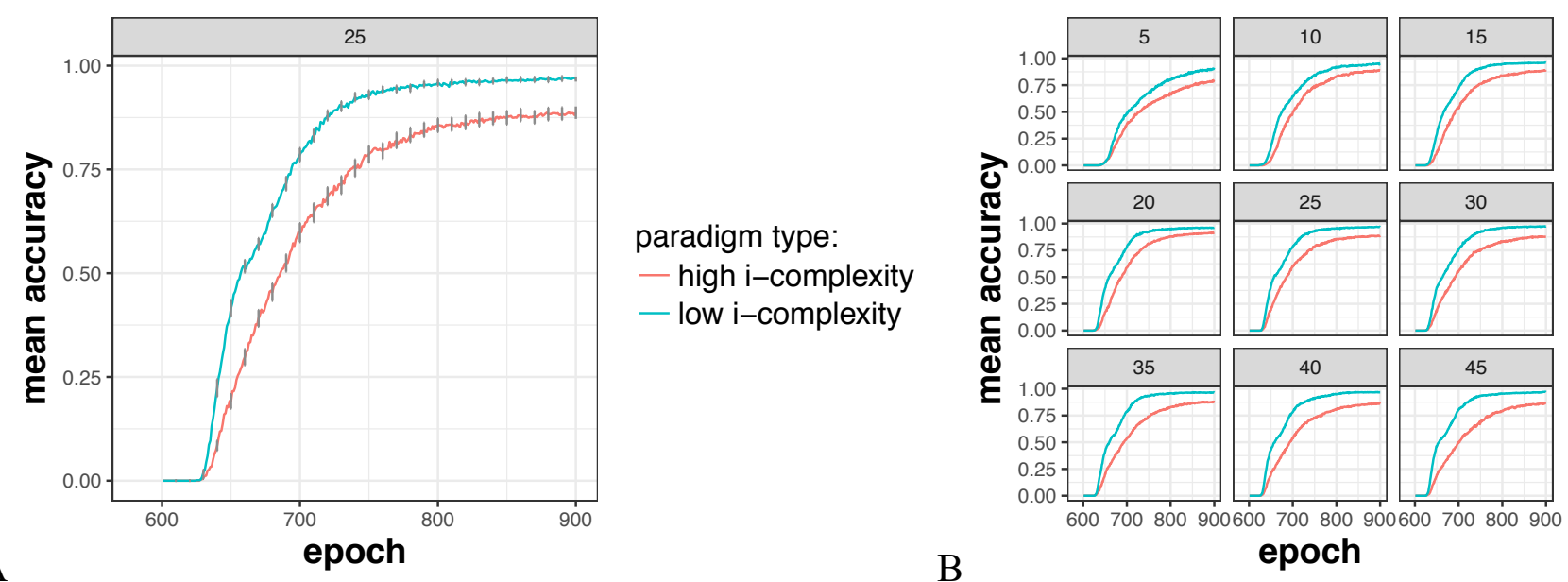

Figure 2. Average accuracy across all runs of the LSTM networks in generalizing to novel dual forms for the low i-complexity paradigm (blue) and the high i-complexity paradigm (red). A: results for one network size ( 25 cells), with error bars indicating standard error every 10 epochs. B: results for all the network sizes tested (facet titles give network size in number of cells). Note that the plots start at epoch 600, when the dual forms are introduced to the network (at the beginning of Block 3). In all cases accuracy is higher for the low i-complexity paradigm. 
These results show that for LSTM networks, low i-complexity facilitates solving the Cell Filling Problem. This is in line with Ackerman and Malouf (2013) and Seyfarth et al. (2014), who focus on this situationin which unseen forms must be generated for a stem-as the critical problem for learners. As discussed above, learning a morphological system involves not only generalizing to completely novel stems, but also learning and retrieving forms for stems which are not yet robustly learned (e.g., due to low exposure frequency). We test this more general facilitative effect of low i-complexity in the next set of simulations.

\section{Simulation Experiment 2 - learning speed}

\section{The model}

We tested LSTM networks with the same architecture, input and output representations, and parameters as described for Simulation Experiment 1. In this simulation, the language includes nine stems. The model was trained and tested in three blocks, summarized in Table 6. In the first block, the network was trained and tested on singulars for all stems; in block 2 the network was trained and tested on singulars and plurals for all stems; in block 3 the network was trained and tested on the entire paradigm for all stems, including the dual. Note that, unlike for Simulation Experiment 1, here the test set is always identical to the training set, i.e. we are not testing on the capacity of the network to generalize, but simply to learn the mapping from stem-number input to the appropriate affix. As before, we tested networks of different sizes, from 5cells networks (542 parameters) to 45 (12,022 parameters), in increments of 5; we conducted 100 runs for each paradigm for each network size, with initial weights of the network randomly generated for each run.

\begin{tabular}{llll}
\hline Block & Epochs & Training & Testing \\
\hline 1 & 300 & all stems, singular only (9 trials) & all stems, singular only (9 trials) \\
2 & 300 & all stems, singular and plural (18 trials) & all stems, singular and plural (18 trials) \\
3 & 300 & all stems, all numbers (27 trials) & all stems, all numbers (27 trials) \\
\hline
\end{tabular}

Table 6: Summary of training and testing blocks for RNNs in Simulation Experiment 2.

\section{Results}

Figure 3 presents the learning trajectories of the neural networks for the singular and plural forms in the low and high i-complexity paradigms. Networks of all sizes show similar learning trajectories and final accuracy levels across both paradigms, as expected since there is no difference between the paradigms for these forms. In all sizes of networks, except for size 5 (Figure 3A), the networks reach perfect learning of the singular and plural forms (accuracy of 1), and the singular and plural forms are fully learned (or near perfect learning) by the beginning of block 3 (epoch 600), when the dual forms are introduced to the network. These results verify that the networks are able to learn the singular and plural forms. This is necessary in order to exploit the predictive structure of the paradigms to better learn the dual. Interestingly, the smaller networks show a small decrement in accuracy of plural learning after epoch 600 , the point at which the dual is introduced; networks of size 5 show some differences between high and low i-complexity in learning the plural forms after that point, with a more rapid recovery for networks trained on the low icomplexity paradigm. 

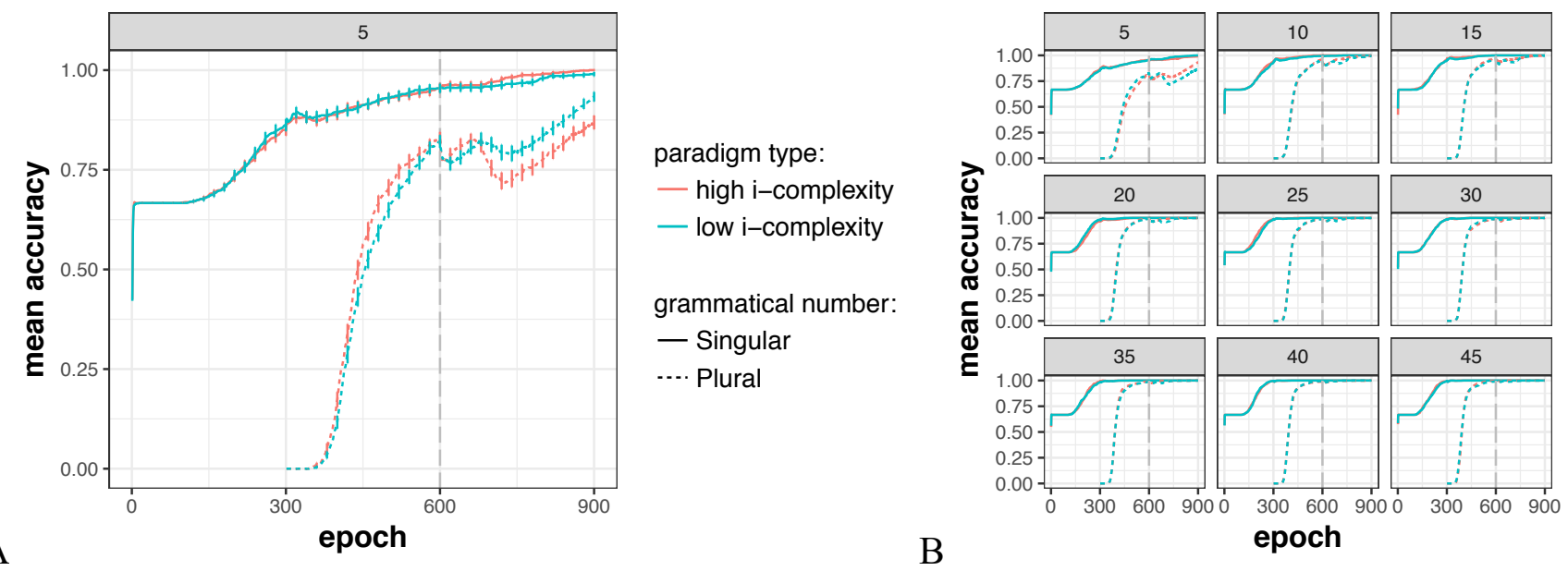

Figure 3. Network learning trajectories for singular and plural forms for high and low i-complexity paradigms. A: results for one network size (5 cells), with error bars indicating standard error every 10 epochs, B: results for all the network sizes tested (facet titles give network size in number of cells). Note that dashed lines for plural suffixes start at epoch 300 (block 2). Networks exposed to the high icomplexity language and low i-complexity language show similar performance.

Figure 4 presents the learning trajectories of the neural networks for the dual forms. Across all network sizes, the dual forms are learned faster in the low i-complexity paradigm. This difference in learning speed for low vs high i-complexity can also be seen in Figure 5, showing for every network size the epoch in which the network reached perfect learning of the full paradigm. These results show that the predictive relationships in low i-complexity paradigms facilitate learning in these networks beyond generalisation to entirely novel stems.
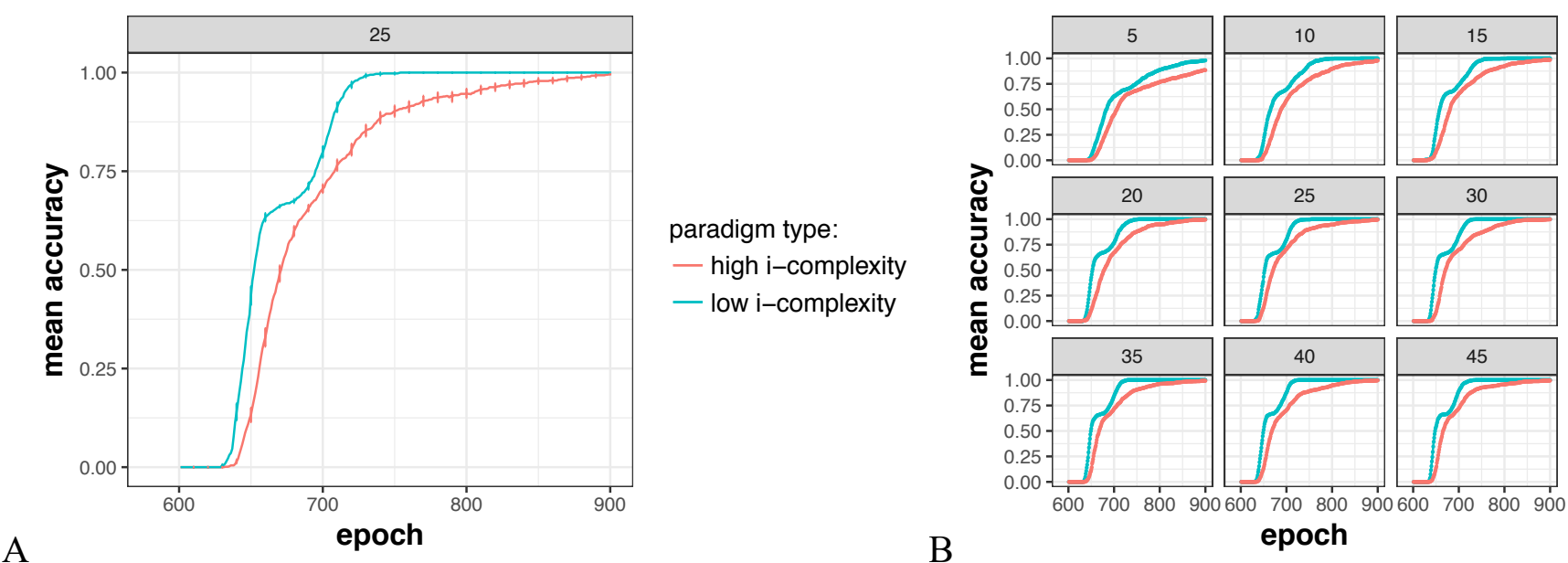

Figure 4. Network learning trajectories for dual forms for high and low i-complexity paradigms. A: results for one network size ( 25 cells), with error bars indicating standard error every 10 epochs, B: results for all the network sizes tested (facet titles give network size in number of cells). Note that the plots start at epoch 600 , when the dual forms are introduced to the network (block 3 ). 


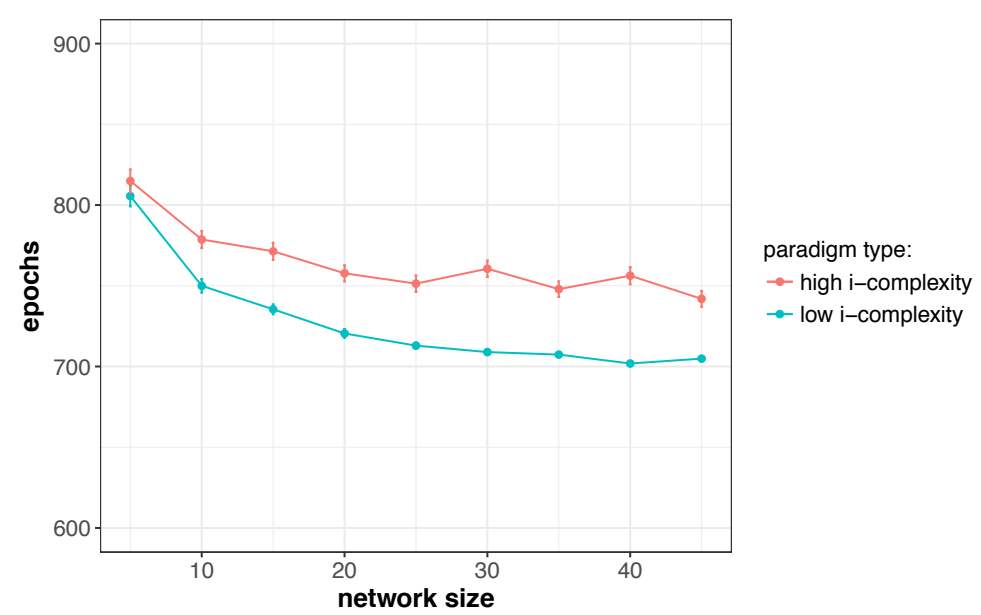

Figure 5. Number of training epochs required to reach perfect learning of the paradigm for each size of network.

Together, results from Simulation Experiments 1 and 2 show that for LSTM neural networks, the icomplexity measure is predictive of learning and generalisation when controlling for other factors, such as number of different markers, e-complexity, and inflection frequency. When trained on inflectional paradigms with low i-complexity, LSTM networks showed higher accuracy in generalizing to completely unseen dual forms, and more rapid learning of dual forms which were trained at low frequency. In principle then, and for at least one learning model, i-complexity influences generalization and learning of inflectional paradigms. This is consistent with Ackerman and Malouf (2013), who claim that morphological learning should be modulated by i-complexity-a measure of the predictive structure of a paradigm. However, we are specifically interested in human learning, since it is biases in human learning that will shape human languages. While RNNs may mimic some features of human learning they cannot fully simulate human learning (e.g., Linzen et al., 2016; Gulordava et al., 2018). Below, we present a series of artificial language learning experiments to test the effects of i-complexity with human participants.

\section{Experiment 1}

As discussed above, Seyfarth et al. (2014) provide some evidence that human learners use the paradigmatic information captured by i-complexity to predict new forms. They found that, in a $2 \times 3$ paradigm, learners used the similarity between novel forms and trained forms to guess inflectional endings. In a slightly larger $3 \times 3$ paradigm, learners used this predictive information to guess low frequency inflectional endings. In the following experiments, we test whether i-complexity affects the speed with which a paradigm is learned, rather than the ability to generalize to completely novel forms. Participants were asked to learn labels for objects through trial and error, where these labels were drawn from one of the two artificially constructed paradigms described above. As in the network simulations, the icomplexity of the language was manipulated between subjects; the only difference between paradigms was the predictability of the dual form, which in the low-complexity language was predictable from the singular. Predictive relationship among forms in a paradigm can in principle facilitate learning of new (initially low-frequency) forms, and indeed neural networks showed precisely this benefit in our Simulation Experiment 2. If i-complexity also mediates human learning in this context, then participants in the low i-complexity condition should show faster learning and higher accuracy in inflecting nouns in the dual after learning the singular and plural forms. 


\section{Materials}

Participants were trained on one of the two paradigms in Table 4 above. While our networks simulations did not involve any referents, here the nine stems referred to a set of simple objects (lemon, cow, tomato, bicycle, horse, clock, pigeon, mug and pear) depicted by photographs. Singular nouns corresponded to a single object, dual corresponded to two of the objects, and plural ranged from 3-12 randomly (see Figure 6). Stems and suffixes were randomly paired with meanings for each participant. As before, stems were randomly allocated to classes. In addition, noun class membership was not conditioned on meanings; every noun class had one animate object (cow/pigeon/horse), one edible object (tomato/lemon/pear) and one other (clock/bicycle/mug).

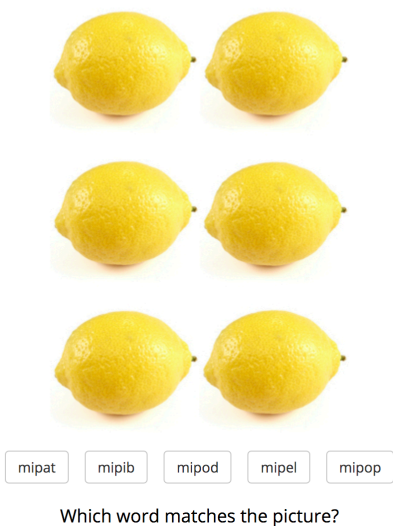
A Figure 6. Example plural trials. A: a picture is presented and participants are asked to choose the correct label from a set of options. B, C: participants receive feedback on their answer, including the correct label. $\mathrm{B}$ : negative feedback following trial shown in $\mathrm{A}, \mathrm{C}$ : positive feedback following plural trial with a different number of objects.

\section{Participants}

Participants were 39 adults recruited via Amazon's Mechanical Turk crowdsourcing platform. They were paid $\$ 4.50$. Participants were allocated randomly to one of the two conditions (20 in the high i-complexity condition, 19 in the low i-complexity condition).

\section{Procedure}

On each trial, a picture was presented on the screen together with a set of possible stem + suffix labels, as in Figure 6A. Participants were asked to choose the correct label, and received feedback on their answer as in Figure 6B,C. The task was divided into 3 blocks of trials. In block 1 (36 trials), participants were exposed to the singular forms of all stems. In block 2 (72 trials) plural trials of all stems were introduced along with singulars. Finally, in the critical block 3 (108 trials), participants were exposed to all stems in all cells of the paradigm, including the dual. Each word form was presented four times in each block of trials. The different forms were randomly interspersed within each block.

\section{Results}

Figure 7 shows the mean accuracy with which participants chose the appropriate word form for singular, plural, and dual, as the experiment progressed trial by trial. Recall that we are particularly interested in how well participants learned the dual forms in block 3 , after being trained on the singular and plural forms. Participants exposed to the high i-complexity language had mean accuracy in dual trials of $M_{H}=0.58(\mathrm{sd}=0.19)$ whereas in the low i-complexity condition mean accuracy was $M_{L}=0.50(\mathrm{sd}=0.23)$. 
To test the effect of i-complexity on production of dual forms, we ran a mixed effects logistic regression model predicting dual accuracy rates by condition (high vs. low i-complexity), accuracy on block 2 , trial number, and their interactions as fixed effects. ${ }^{4}$ Trial number was scaled and centered such that estimates for the effect of condition reflect the difference between conditions mid-way through block 3 . Condition was sum-coded (high i-complexity $=-1$, low i-complexity $=1$ ). We included participants' accuracy in block 2 as a way of controlling for general differences in learning ability. This is crucial, since participants in the high i-complexity condition were actually more accurate in blocks 1 and 2 , despite no difference in the training participants had received at this point in the experiment. ${ }^{5}$ Accuracy at block 2 was centered and scaled such that estimates for the effect of condition reflect the difference between conditions for participants with average accuracy in block 2 . The model revealed a significant interaction between accuracy in block 2 and trial number $(b=1.387, \mathrm{z}=2.856, \mathrm{p}=0.004)$, indicating that participants who learned the singulars and plurals better in block 2 learned the duals more rapidly in block 3 . Results from the model also show significant effect for trial number $(b=2.05, \mathrm{z}=4.44, \mathrm{p}<0.001)$, showing that participants' accuracy across conditions improved over trials. Crucially however, there was no significant effect of condition $(\mathrm{b}=0.11, \mathrm{z}=0.72, \mathrm{p}=0.471)$ on dual accuracy rates, and no significant interaction between condition and trial number $(\mathrm{b}=0.047, \mathrm{z}=0.295, \mathrm{p}=0.768)$.

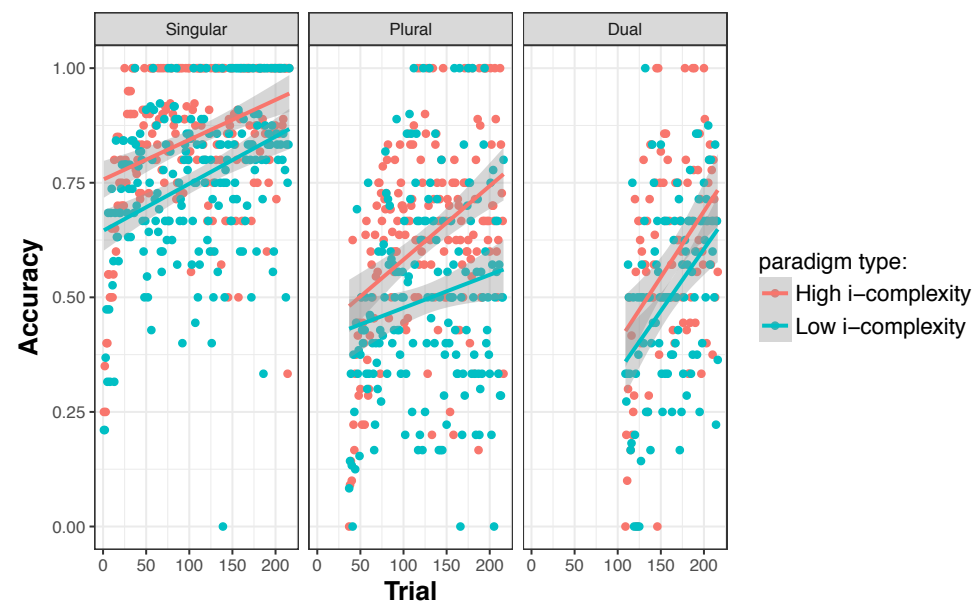

Figure 7. Mean accuracy by trial for singular, plural, and dual forms. Points indicates participants' mean accuracy scores in the low and high i-complexity conditions, with a regression line predicting accuracy by trial number for each grammatical number. Participants in the high i-complexity condition (unexpectedly) showed better accuracy in blocks 1 and 2 . When controlling for accuracy in block 2, there was no significant effect of condition on dual learning.

Our results suggest that there is no learnability advantage for the low i-complexity paradigm; numerically our participants exposed to high i-complexity paradigms actually learned more quickly, but our statistical analysis shows that any apparent difference in dual accuracy across the two conditions in Figure 7 is in fact driven by individual differences in learning ability/attentiveness, rather than the structure of the target paradigm. This experiment therefore fails to confirm the predicted learning advantage for lower icomplexity paradigms. There are at least three possible explanations for why we fail to find this predicted advantage in our task. One possibility is that, for human learners, lower i-complexity provides some advantage in generalizing to completely novel words (Seyfarth et al., 2014), but does not continue to

\footnotetext{
${ }^{4}$ All models reported here were run using the lme4 package in R (Bates, Meachler, Bolker \& Walker, 2015). All models include byparticipant intercepts and random slopes for trial number.

${ }^{5}$ This was confirmed by a mixed-effects logistic regression model predicting accuracy in block 2 by condition and trial number (high vs. low i-complexity: $b=-0.268, z=0.122, p=0.028)$.
} 
facilitate learning of forms already encountered, even in low frequency. Here, it is also possible to learn the forms through memorization, ignoring any predictive structure in the paradigm that might be helpful. Another possibility is that we don't see the effect i-complexity for methodological reasons; either because we don't have enough critical (dual) trials to reveal differences between the two conditions, or, because the predictive relationship from one form to another was not readily accessible to participants. In our task, the different forms of the nouns were randomly interspersed within each block, while learners might need a more explicit cue to the implicative structure of the paradigm. Studies in first language acquisition suggest that variation sets in the input to children, pairs of utterances that balance their overlap and change facilitate vocabulary growth (e.g., Waterfall, 2005; Brodsky, Waterfall \& Edelman, 2007; Tal \& Arnon, 2018). In a similar way, presenting the new forms in the language after presenting a familiar form of the same noun could make the predictive structure of the paradigm more apparent for learners. In Experiments 2-3, we therefore added more critical trials (a $4^{\text {th }}$ block) and structured trials to highlight the predictive structure of the paradigms. In particular, we organized the trials in blocks 3-4 in pairs, so that dual trials always followed either plural or singular trials, with the same object. This design is parallel to the task Seyfarth et al., (2014) used to test generalization to novel forms, but note that here we are always presenting and testing on familiar nouns.

\section{Experiment 2}

\section{Materials}

All materials were identical to Experiment 1.

\section{Participants}

Participants were 41 adults recruited via Amazon Mechanical Turk. They were paid \$6. Participants were allocated randomly to one of the conditions (20 high i-complexity, 21 low i-complexity).

\section{Procedure}

The procedure was identical to Experiment 1 with two exceptions. First, the task included 4 blocks of trials, where block 4 was identical in its structure to block 3. Second, blocks 3 and 4 included all word forms in the paradigm, set up in pairs: singular or plural trial were always immediately followed by a trial with the same object in a different number, as illustrated in Figure 8. Critically, when the following trial was dual, the predictive information in the low i-complexity paradigm could be particularly helpful to learners. This arrangement resulted in three types of pairs: singular trial followed by a dual trial ('predictive trials'), plural followed by dual trial and singular followed by plural. The number of each type of pair was balanced and different pairs of trials were randomly interleaved. 


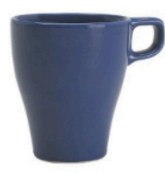

mipod mipum mipop mipoc mipat mipel mipib

A

Which word matches the picture?

Figure 8. Example of two successive trials in blocks 3 and 4 in Experiment 2. A: trial $n$, in which participant is asked to choose the correct form describing a mug in singular, B: trial $n+1$, in which participant is asked to choose the correct label for the same object in dual.

\section{Results}

Figure 9 shows the mean accuracy with which participants chose the appropriate word form for singular, plural, and dual, as the experiment progressed trial by trial. Mean accuracy on the critical dual trials is higher for the low i-complexity condition $\left(M_{L}=0.71\right.$, sd $\left.=0.23\right)$ than for the high i-complexity condition $\left(M_{H}=0.55, \mathrm{sd}=0.26\right)$. However, as in Experiment 1 , differences between conditions already appeared in blocks 1 and 2, despite the fact that the input languages are identical up to this point. ${ }^{6}$ We ran a mixed-effects logistic regression model predicting dual accuracy rates by condition (high vs. low icomplexity), accuracy on block 2, trial number and their interactions as fixed effects, with fixed effects coded as in Experiment 1. Results again revealed a significant effect of trial number $(b=1.45, z=7.81$, $\mathrm{p}<0.001)$ and a significant interaction between accuracy in block 2 and trial number $(b=0.66, z=0.193$, $\mathrm{p}<0.001)$, but no significant effect of condition $(b=0.141, \mathrm{z}=0.175, \mathrm{p}=0.42)$, and no condition by trial number interaction $(b=0.223, z=0.178, p=0.211)$. These results hold when looking only at predictive trials (dual trials following singular trials): the interaction of accuracy in block 2 with trial number is significant $(b=0.652, z=3.1, p=0.001)$ and so is the effect of trial number $(b=1.09, z=5.7, p<0.001)$, but there is no main effect of condition $(b=0.142, z=0.87, p=0.383)$, and no interaction between condition and trial number $(b=0.191, z=1.03, p=0.301)$. To summarize, lower $\mathrm{i}$-complexity did not lead to a learning advantage when controlling for participants' earlier learning, not even in predictive trials, where the singular form which predicts the dual is easily accessible.

\footnotetext{
${ }^{6}$ This was confirmed by a mixed-effects logistic regression model predicting accuracy in block 2 by condition and trial number (high vs.
ow i-complexity: $b=0.314, z=2.395, p=0.016)$. In this case, participants allocated to the low i-complexity condition were significantly better

${ }^{6}$ This was confirmed by a mixed-effects logistic regression model predicting accuracy in block 2 by condition and trial number (high vs.
low i-complexity: $b=0.314, z=2.395, p=0.016$ ). In this case, participants allocated to the low i-complexity condition were significantly better in block 2 .
} 


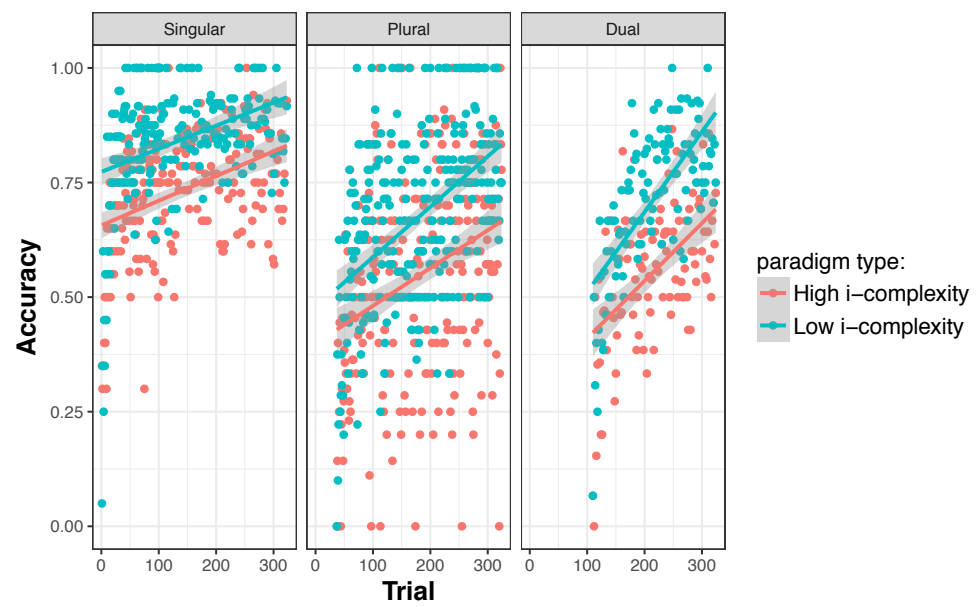

Figure 9. Mean accuracy by trial for singular, plural, and dual forms in Experiment 2 (with predictive trials). Points indicates participants' mean accuracy scores in the low and high i-complexity conditions, with a regression line predicting accuracy by trial number for each grammatical number. Participants in the low i-complexity condition (unexpectedly) showed better accuracy in blocks 1 and 2 . When controlling for accuracy in block 2 , there was no significant effect of condition on dual learning.

\section{Experiment 3}

While Experiments 1 and 2 are consistent in showing no advantage for low i-complexity, our confidence in this conclusion was reduced by the substantial inter-individual differences in our sample; in both Experiments we found substantial differences between conditions in learning accuracy in block 2, before the conditions had diverged. In Experiment 3 we replicate Experiment 2 with more participants in order to reduce the likelihood of obtaining samples with accidental differences between the two conditions; since Experiment 3 uses an identical method to Experiment 2, a combined analysis (total $\mathrm{N}=100$ ) also becomes possible.

\section{Participants}

Participants were 59 adults recruited via Amazon Mechanical Turk. They were paid \$6. Participants were allocated randomly to one of the conditions (30 high i-complexity, 29 low i-complexity).

\section{Materials and Procedure}

This experiment was identical to Experiment 2.

\section{Results}

Figure 10 shows the mean accuracy with which participants chose the appropriate word form for singular, plural, and dual, as the experiment progressed trial by trial. In this case, learners in both conditions were more balanced with respect to their general ability to learn in the task. ${ }^{7}$ Mean accuracy in dual trials was higher in the low i-complexity condition $\left(M_{L}=0.69, \mathrm{sd}=0.25\right)$ than in the high i-complexity condition $\left(M_{H}=0.55, \mathrm{sd}=0.25\right)$. We used a mixed-effects logistic regression model, as in Experiments 1-2, to predict dual accuracy based on condition (high vs. low i-complexity), accuracy on block 2, and trial number. The model revealed a significant effect of condition $(b=0.328, z=2.208, p=0.027)$, as well as a significant interaction between accuracy in block 2 and trial number $(b=0.85, z=5.29, p<0.001)$. The interaction between condition and trial number was not significant $(b=0.189, z=1.26, p=0.205)$. This pattern of results

\footnotetext{
${ }^{7}$ This was confirmed by a mixed-effects logistic regression model predicting accuracy in block 2 by condition and trial number (high vs. low i-complexity: $b=0.176, z=1.47, p=0.141$ ). In other words, there was no significant difference between learners in the two conditions.
} 
suggests that, as in Experiments 1 and 2, participants who showed better learning in block 2 also showed faster learning of the dual forms. However, in addition, participants in the low i-complexity group were better at learning the dual forms; in other words, there was a learning advantage for participants in the low i-complexity condition, when controlling for individual differences in learning abilities. These effects also hold when looking only at predictive trials: the main effect of condition is significant $(b=0.33, z=2.00$, $\mathrm{p}=0.045)$, although the interaction of condition with trial number is not $(b=0.12, z=0.722, p=0.47)$.

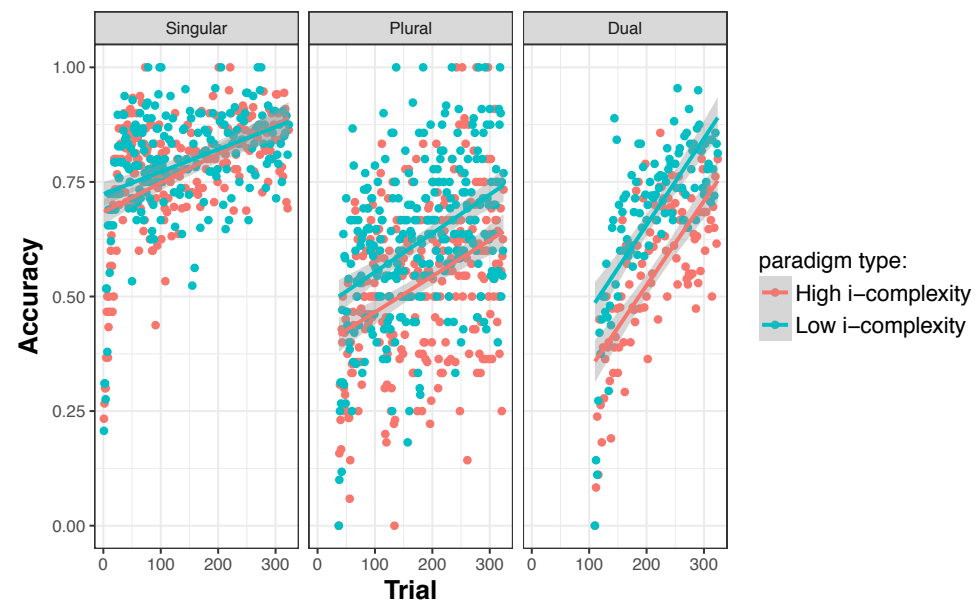

Figure 10. Mean accuracy by trial for singular, plural, and dual forms in Experiment 3 (replication with predictive trials). Points indicates participants' mean accuracy scores in the low and high i-complexity conditions, with a regression line predicting accuracy by trial number for each grammatical number. Participants were well matched in blocks 1 and 2. When controlling for accuracy in block 2, participants in the low i-complexity condition were better in learning the dual forms.

Since Experiments 2 and 3 were identical, we also ran a combined analysis including both data sets. We ran a mixed-effects logistic regression model, as before, predicting accuracy on dual trials from condition, experiment, trial number, and accuracy in block 2 . The model revealed a significant effect of condition $(b=0.236, z=2.012, p=0.044)$ as well as a significant interaction between accuracy in block 2 and trial number $(b=0.7625, z=5.924, p<0.001)$. The interaction of condition and trial number was marginally significant $(b=0.214, \mathrm{z}=1.81, \mathrm{p}=0.070)$, and there was no significant effect of experiment $(b=0.04$, $\mathrm{z}=0.342, \mathrm{p}=0.732$ ). The results suggest that, after correcting for the random imbalance in learners across the two conditions, we see some evidence of an advantage for the low i-complexity language, both in higher overall performance and (marginally) faster learning.

To test whether the effect of condition found in Experiments 2 and 3 was directly related to the presence of predictive trials, we also ran an additional analysis comparing data across all three experiments. Recall that in the predictive trials design used in Experiments 2-3, participants were asked for the dual form immediately after getting the singular form in the previous trial. If learners only make use of the predictive information when they have ready access to a predictive form, these trials should facilitate learning of the dual forms, but only in the low i-complexity conditions of Experiments 2 and 3 , not Experiment 1. We ran a mixed-effects logistic regression predicting accuracy on dual trials from experiment, condition, accuracy in block 2, and trial number. Coding for condition and trial number was the same as in previous analyses. Experiment was sum coded (Experiment $1=-1$, Experiments 2 and $3=$ 1). Trials from block 4 in Experiments 2 and 3 were excluded from the analysis, to match number of blocks and trials across the three Experiments. A benefit for predictive trials should manifest as an interaction between experiment and condition in this model. The model revealed a significant interaction 
between trial number and experiment $(b=0.265, z=2.63, p=0.008)$, but no significant interaction between experiment and condition $(b=0.067, z=0.716, p=0.474)$ and no significant effect of condition $(b=1.43$, $\mathrm{z}=1.527, \mathrm{p}=0.12$ ). This suggests that the predictive trials design was actually beneficial for participants in both conditions; being exposed to one form of a noun helped participants to subsequently retrieve the dual, regardless of the i-complexity of the paradigm. This is in line with studies showing that repetition of stems in different contexts (sometimes called overlap) facilitates word learning in first language acquisition (Waterfall, 2005; Brodsky, Waterfall \& Edelman, 2007; Tal \& Arnon, 2018). However, there is no evidence that the predictive trials provided any particular advantage for participants in the low icomplexity condition, for example by making the predictive link between forms in the low i-complexity paradigm more apparent to learners.

At this point, our results show only quite weak evidence for effects of i-complexity on learning inflectional paradigms. On the one hand, we see a significant effect of i-complexity on learning in one of our experiments (Experiment 3) and in the combined analysis of 100 participants across Experiments 2 and 3 , in which we used the same method, namely the predictive trials in blocks 3 and 4 of the experiments. However, Experiments 1 and 2 when considered independently do not show a significant effect of icomplexity on learning, and a combined analysis of all three experiments (combined $\mathrm{N}=139$ ) does not show a significant effect of i-complexity or that the predictive trials method interacts with i-complexity.

\section{Testing the impact of e-complexity on paradigm learning}

Given the rather weak evidence of an effect of i-complexity for human learners, we attempted to test whether another measure of paradigm complexity (e-complexity) had a more robust effect; recall that natural language paradigms differ quite substantially on e-complexity. Using the simulation and experimental methods above, we manipulate e-complexity to test its effect on paradigm learning in RNNs and human participants and to compare the effect this measure has on learning with that of i-complexity. The paradigms used in Simulation Experiments 1-2 and Experiments 1-3 varied in i-complexity but had low e-complexity. We constructed a third inflectional paradigm similar in size and design to the ones used above, with high e-complexity and low i-complexity (Table 7) and tested how well RNNs and human participants learned the forms in the paradigm. In Simulation Experiment 3 we compare results from RNNs learning the paradigm with results from Simulation Experiment 2. In Experiment 4 we compare results from human participants learning the high e-complexity paradigm with combined data from Experiments 2 and 3. This allows us to compare the effect that the e-complexity of the paradigm has on its learnability with the effect of i-complexity.

\section{High e-complexity paradigm}

In this Experiment, RNNs and participants are trained and tested on an inflectional paradigm of an artificial language. Higher e-complexity is reflected by having more variants to mark the dual forms. We use the same paradigm in the simulation experiment with RNNs and in the experiment with human participants.

\begin{tabular}{lccc}
\hline & Singular & Dual & Plural \\
\hline noun class 1 & - at & $-o c$ & $-a t$ \\
noun class 2 & $-o p$ & $-u m$ & $-e l$ \\
noun class 3 & -at & $-\mathrm{ib}$ & -od \\
\hline
\end{tabular}

Table 7. Example paradigm for high e-complexity language. This paradigm is of the same size as in previous experiments ( 3 noun classes, 3 grammatical numbers, 7 inflections), but with higher e-complexity (Table 7). The high e-complexity paradigm has higher e-complexity than the paradigms shown in Table $4,1.36$ vs. 1.14 bits, while its overall i-complexity is equal to that of the low i-complexity paradigm in Table 4A, 0.222 bits. The higher e-complexity is due to an additional variant to mark the dual; we keep 
the overall number of inflections constant by re-using one marker from the singular in the plural (in this example, -at).

\section{Simulation Experiment 3}

\section{The model}

We tested LSTM networks with the same architecture, input encoding and parameters as described for Simulation Experiments 1 and 2. Training and testing the network on the forms according to the paradigm in Table 7 followed the same procedure as in Simulation Experiment 2, summarized in Table 6. As before, we tested networks of different sizes, from 5-cells networks (542 parameters) to 45 (12,022 parameters), in increments of 5; we conducted 100 runs for each paradigm for each network size, with initial weights of the network randomly generated for each run.

\section{Results}

Figure 11 presents the learning trajectories of the neural networks for the dual forms, comparing the results from networks trained on the paradigm with new high e-complexity with results from Simulation Experiment 2. We designate the two paradigms from Table 4 as low i-complexity and high i-complexity; note that both these paradigms have lower e-complexity compared to the new paradigm in Table 7; our high e-complexity paradigm has the same i-complexity as the low i-complexity paradigm in Table 4A. Across all network sizes, the dual forms in the high e-complexity paradigm are learned slower than both the low i-complexity and high i-complexity paradigms. This difference in learning speed can also be seen in Figure 12, showing for every network size the epoch in which the network reached perfect learning of the full paradigm.

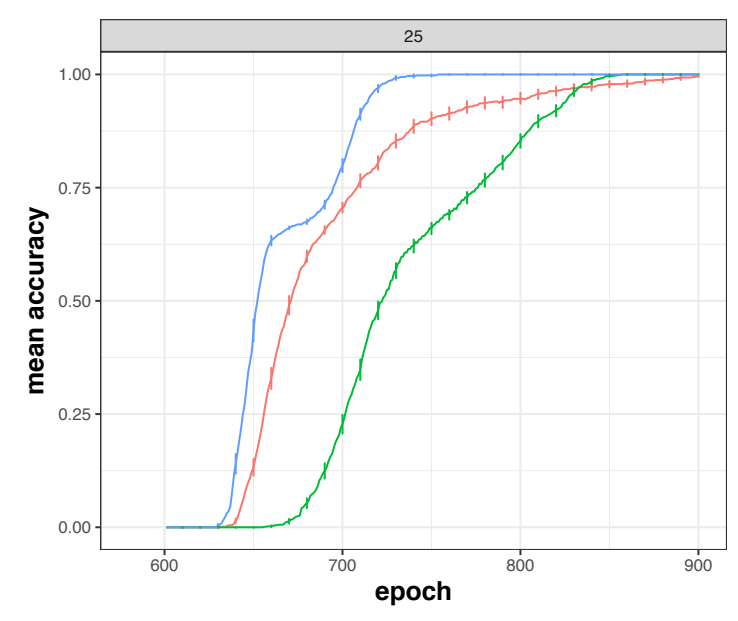

A

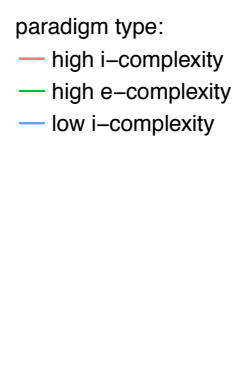

Figure 11. Average accuracy across all runs of the LSTM networks in generalizing to novel dual forms for the new high e-complexity paradigm (green) compared with results from the low i-complexity paradigm (blue) and the high i-complexity paradigm (red) from Simulation Experiment 2. A: results for one network size ( 25 cells), with error bars indicating standard error every 10 epochs. B: results for all the network sizes tested (facet titles give network size in number of cells). Note that the plots start at epoch 600 , when the dual forms are introduced to the network (at the beginning of Block 3 ). In all cases accuracy for the high e-complexity paradigm is lower than for both other paradigms. 


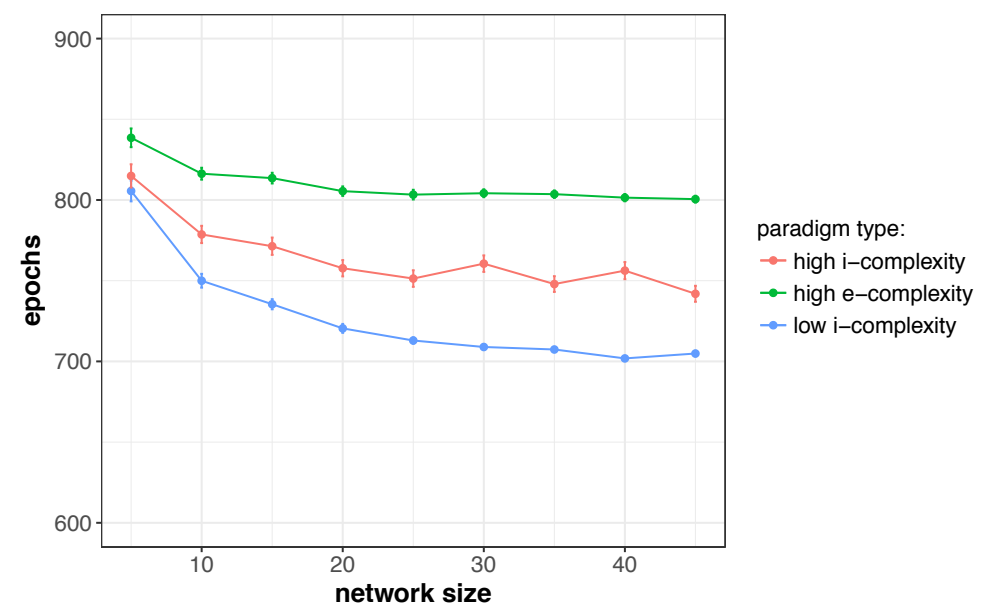

Figure 12. Number of training epochs required to reach perfect learning of the paradigm for each size of network.

These results suggest that for LSTM neural networks, while the i-complexity measure is predictive of learning the forms in the paradigm when controlling for e-complexity (as we showed in Simulation Experiment 2), the measure of e-complexity is more dominant in its effect on learning: the new high ecomplexity paradigm is learnt more slowly than either of our low e-complexity paradigms, even though it has low i-complexity.

\section{Experiment 4}

The results from Simulation Experiment 4 suggest that for LSTMs e-complexity has more of an effect on learning than i-complexity, Next, we test the effect of e-complexity on learning inflectional paradigms in human learners, and compare it with the effect of i-complexity. We use the combined data from Experiments 2-3 to represent paradigms with low e-complexity but varying i-complexity.

\section{Participants}

Participants were 50 adults recruited via Amazon Mechanical Turk. They were paid \$6. All participants were allocated to the high e-complexity condition.

\section{Materials and Procedure}

This experiment was identical to Experiments 2 and 3, with the sole difference of training participants on an inflectional paradigm with high e-complexity (Table 7).

\section{Results}

Figure 13 shows the mean accuracy with which participants chose the appropriate word form for singular, plural, and dual, as the experiment progressed trial by trial. Results from this experiment are compared with data from Experiments 2 and 3, where participants were exposed to paradigms with low e-complexity and either low or high i-complexity. Participants exposed to the high e-complexity language had mean accuracy in dual trials of $M_{\text {high }}=0.45(\mathrm{sd}=0.25)$ whereas in the low e-complexity paradigms participants mean accuracy was $M_{l o w}=0.69(\mathrm{sd}=0.24)$ (in the low i-complexity condition) and $M_{\text {high } i}=0.56(\mathrm{sd}=0.26)$ (in the high i-complexity condition). 
There was no significant differences between conditions in blocks 1 and 2, in which the linguistic input was identical for all conditions. ${ }^{8}$ To test the effect of e-complexity on production of dual forms and to compare it with the effect of i-complexity, we ran a mixed-effects logistic regression model predicting dual accuracy rates by condition (low i-complexity [with low e-complexity], high i-complexity [with low e-complexity], and high e-complexity [with low i-complexity]), accuracy on block 2, trial number and their interactions as fixed effects. Condition was dummy-coded with high e-complexity as the reference level, to compare the other two conditions to it. Other fixed effects were coded as in Experiments 1 and 2. The results of this experiment closely mirror the findings of Simulation Experiment 3. Results revealed a significant effect of e-complexity, with higher accuracy for the low i-complexity condition than the high e-complexity condition $(b=1.502, z=7.3, p<0.001)$, and significant interaction between e-complexity and trial number $(b=0.67, z=3.21, p=0.0013)$, suggesting that participants in the low i-complexity, low ecomplexity condition improved faster in learning the dual forms than participants learning the new high e-complexity, low i-complexity paradigm. Accuracy in the high e-complexity condition was also significantly lower than in the high $\mathrm{i}$-complexity, low e-complexity condition $(\mathrm{b}=0.99, \mathrm{z}=0.2, \mathrm{p}<0.001)$, but in this case there was no significant interaction between condition and trial number $(b=0.299, z=1.46$, $\mathrm{p}=0.14)$. As seen in earlier experiments there were also significant effects of trial number $(\mathrm{b}=1.04, \mathrm{z}=7.44$, $\mathrm{p}<0.001)$, accuracy in block $2(\mathrm{~b}=1.3, \mathrm{z}=8.26, \mathrm{p}<0.001)$, and a significant interaction between accuracy in block 2 and trial number $(b=0.52, z=3.21, p=0.0013)$.

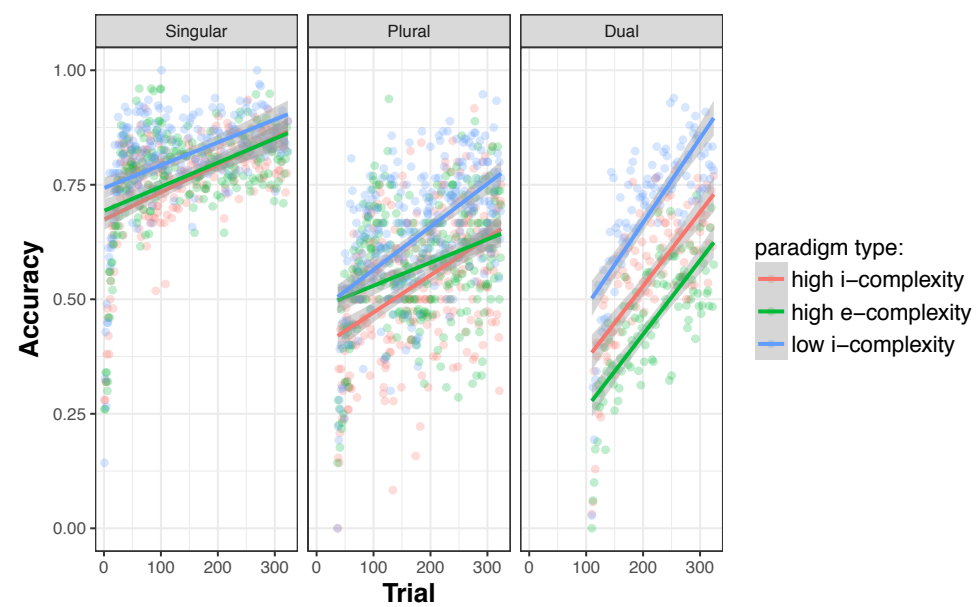

Figure 13. Mean accuracy by trial for singular, plural, and dual forms of participants exposed to the high e-complexity paradigm (in green) compared with results from Experiments 2 and 3 where participants were exposed to paradigms with low e-complexity and either low or high i-complexity. When controlling for accuracy in block 2, participants in the high e-complexity condition were worse in learning the dual forms than both low e-complexity paradigms.

To summarise, the results of experiments with both neural network and human participants suggest that when comparing the effect of e-complexity with the effect of i-complexity on learning, e-complexity has a stronger effect; a paradigm with high e-complexity but low i-complexity is learnt more slowly than paradigms with lower e-complexity, regardless of whether those paradigms have the same or higher icomplexity.

\footnotetext{
${ }^{8}$ This was confirmed by a mixed-effects logistic regression models predicting accuracy in block 2 by condition and trial number (high vs. low e-complexity: $b=0.167, z=0.97, p=0.33$; high i-complexity vs. high e-complexity: $b=-0.29, z=-1.7, p=0.088$ ).
} 


\section{Discussion}

We used neural network simulations and behavioral experiments with humans to test whether the learnability of artificially-constructed inflectional paradigms was predicted by i-complexity. This information-theoretic measure of complexity, proposed by Ackerman and Malouf (2013), captures the extent to which forms in a paradigm predict each other. We tested whether a paradigm in which dual forms could be predicted from singular forms (lower i-complexity) was learned faster than a paradigm in which the dual is less well predicted from the singular (higher i-complexity), holding other measures of complexity constant. In contrast with previous work, we tested not just the ability to generalize to completely novel forms, but also the speed and overall accuracy with which the paradigms were learned. Results from network simulations with LSTMs showed that low i-complexity was advantageous both for generalizing the paradigm to novel forms (i.e., solving the Cell Filling Problem, Ackerman \& Malouf, 2009) and early learning of new forms that have not yet been robustly acquired (i.e., through memorization). These findings show that for an ideal learner, such as an LSTM neural network, the implicative structure of a morphological paradigm, captured by low values of i-complexity, facilitates learning as predicted by Ackerman and Malouf (2013). However, since we are interested here in whether i-complexity shapes cross-linguistic variation in inflectional paradigms through its effect on learnability, it is crucial to verify the effect of i-complexity on human learners.

In previous work, Seyfarth et al. (2014) found evidence that learners use predictive structure to generalize to novel stems. In larger paradigms, with three inflectional classes each expressing three number features, predictive structure was at least partly over-ridden by the effect of inflection frequency: learners only used predictive structure to generate low frequency inflectional forms. Here, we tested whether lower i-complexity paradigms also led to better or faster learning. In Experiment 1, we found no evidence of a learning advantage for our lower i-complexity paradigm when controlling for general differences in learning ability. In Experiment 2, we attempted to reveal an effect of i-complexity by adding more critical trials, and by structuring trials such that a predictive form presented on one trial was followed the cell it predicted on the subsequent trial. In our case, this meant dual trials immediately followed singular trials for the same noun, a trial structure similar in spirit to that used by Seyfarth et al. (2014); structuring the task in this way in principle makes the predictive link between forms in the paradigm more apparent. As in Experiment 1 however, no difference between conditions was found after controlling for general learning ability. In Experiment 3 we replicated this with a larger sample size, and here found some evidence that participants were better-able to learn lower i-complexity paradigms. These results held in a combined analysis of data from Experiments 2 and 3 together. An analysis across all experiments revealed however that participants in both conditions benefited from our constrained ordering of trials. This suggests that there is no critical difference between the experiments with or without the predictive trials ordering in terms of the effect of i-complexity on learning. Further, in this combined analysis there was no evidence that i-complexity modulated learning. Across all three experiments, our results therefore suggest that implicative structure in paradigms provides at best only a weak benefit for human learners.

That said, it is worth noting that the differences in i-complexity between our low- and highcomplexity paradigms were not very large - the difference is 0.222 bits. It could be that larger differences in i-complexity values would reveal a larger effect on learning. However even this difference corresponds to complete predictability of the dual given the singular in the low complexity paradigm, vs at best $66 \%$ predictability in the high complexity paradigm. Testing more extreme values of i-complexity is in principle possible, but would necessitate training participants on much larger inflectional paradigms.

Alternatively, it may simply be that implicative structure is used for generalization to completely novel items, but not once learners have encountered items, even if with low frequency. However, this seems an unlikely explanation as that would presume perfect memory of which items have been encountered. Furthermore, as Seyfarth et al. (2014) show in their study, even when generalizing to novel 
items the effect of the predictive structure is secondary to inflection frequency (a feature better described with measures such as e-complexity). If so, the role of i-complexity in shaping natural language paradigms might be relatively weak. For example, measures of morphological complexity which take into account paradigm size (e.g. number of inflectional classes) and frequency of inflectional forms, e.g., e-complexity, may in fact play a larger role.

In Simulation Experiment 3 and Experiment 4, we test the effect of e-complexity on learning inflectional paradigms to calibrate our understanding of the size of the i-complexity effect by seeing how it compares to an effect of e-complexity. We trained and tested RNNs and human participants on an inflectional paradigm with high e-complexity and low i-complexity. Results from both the simulation with RNNs and the experiment with human participants show a stronger effect on learning of e-complexity than i-complexity; our paradigm with high e-complexity was learned more slowly than the other paradigms we tested, even though it had low i-complexity. These results show that e-complexity is a better predictor of the learnability of an inflectional paradigm. Therefore, the e-complexity or a combination of predictors of learning, rather than i-complexity alone, should be used to explain how learning biases shape morphological paradigms in natural languages.

It is worth noting that our results have implications not just for the specific measure of i-complexity proposed by Ackerma \& Malouf (2013), but also alternative ways of defining the integrative complexity of a morphological system. For example, Sagot (2013) propose to integrate paradigm size into a measure of i-complexity. However, this measure would still predict an effect of i-complexity for our size-matched paradigms. Similarly, Bonamie and Beniamine (2016) propose a version of i-complexity that measures the entropy when predicting an unknown word form based on two other inflections of the same lexeme rather than one. In our study, participants were exposed to two forms of the lexemes (singular and dual) before seeing their forms in dual. Since the plural forms were equally predictive of the dual in both languages, measuring the predictivity of the dual forms using this measure would simply result in a more dramatic difference in complexity across our paradigms (it would make our high i-complexity paradigm even more complex relative to the low i-complexity paradigm).

Our results show that e-complexity is a more robust predictor of morphological learning than icomplexity. However, previous studies suggest that there are differences between adult and children learning (e.g., Hudson Kam \& Newport, 2005, 2009; Culbertson \& Newport, 2015). Since we did not test children learning in our study, it may be that either there is a difference between L1 and L2 learning with respect to the effect of i-complexity, or that e-complexity is more dominant also in first learning acquisition. Future studies should look at the effect of i-complexity in children learning of morphological paradigms.

Furthermore, we show only weak evidence that differences in the reliability of analogy based on distributional cues affects the learning of morphological paradigms. It is however important to note that our results say nothing about using e.g. semantic or phonological similarities to generalize the paradigm to novel words. In the artificial language used here, class membership of lexical items was not conditioned on semantics or phonology; this was crucial to directly test the effect of the predictive structure on learning the inflectional forms independently from any reliance on other cues in predicting inflectional class membership (e.g., Frigo and McDonald, 1998; Gerken et al., 2009; Culbertson et al., 2017). Therefore, our results do not contradict evidence showing that learners use phonological similarities to generalize to new inflections (e.g., Milin, Keuleers \& Đurdevic', 2011; Ambridge, 2010); indeed, one possible future avenue of research is to test whether semantic or phonological cues interacting with distributional information might reveal advantages for low i-complexity paradigms.

\section{Conclusion}


This paper was set to test Ackerman and Malouf's (2013) hypothesis that i-complexity predicts the learnability of morphological paradigms. Using artificial language methods, we tested whether paradigms with low vs. high i-complexity are easier to learn, both with RNNs and with human participants. Results from this study show that while low i-complexity was shown to be beneficial for LSTM neural networks, it is not a strong predictor of learning in human learners; manipulating e-complexity shows a larger effect on learning both in LSTM networks and in human participants, a result which we replicate elsewhere (Johnson, Gao, Culbertson, Rabagliati \& Smith, submitted).

. Together, these results challenge Ackerman and Malouf's hypothesis that i-complexity has a role in shaping paradigms in natural languages. The mismatch between the effect of i-complexity on learning in LSTM networks and in human participants calls for future studies on the different mechanisms of these two learners in learning morphological paradigms. This is of high relevance since more and more studies are proposing ways of measuring morphological complexity and modelling morphological learning using neural networks (e.g. Malouf, 2017; Marzi et al., 2018; Cotterell et al., 2019; Elsner, Sims et al., 2019).

\section{References}

Ackerman, F., Blevins, J. P., \& Malouf, R. (2009). Parts and wholes: Patterns of relatedness in complex morphological systems and why they matter. Analogy in grammar: Form and acquisition, 54-82.

Ackerman, F., \& Malouf, R. (2013) Morphological organization: the low conditional entropy conjecture. Language, 89, 3, 429-464.

Ambridge, B. (2010). Children's judgments of regular and irregular novel past-tense forms: New data on the English past-tense debate. Developmental Psychology, 46(6), 1497.

Aronoff, M. (1994). Morphology by itself: Stems and inflectional classes (No. 22). MIT press.

Baerman, M., Brown, D., \& Corbett, G. G. (2010). Morphological complexity: a typological perspective. Online: http://www. morphology. surrey. ac. uk/Papers/Morphological_complexity. pdf.

Bates, D., Maechler, M., Bolker, B., \& Walker, S. (2015). Fitting Linear Mixed-Effects Models Using lme4. Journal of Statistical Software, 67(1), 1-48.

Bickel, B., \& Nichols, J. (2007). Inflectional morphology. Language typology and syntactic description, 3(2), 169-240.

Blevins, J. P. (2006). Word-based morphology. Journal of Linguistics, 42(3), 531-573.

Blevins, J. P., Ackerman, F., \& Malouf, R. Word and Paradigm Morphology. In The Oxford Handbook of Morphological Theory.

Bonami, O., \& Beniamine, S. (2016). Joint predictiveness in inflectional paradigms. Word Structure, 9(2), 156-182.

Brodsky, P., Waterfall, H., \& Edelman, S. (2007). Characterizing motherese: on the computational structure of child-directed language. In Proceedings of the Cognitive Science Society, 29(29).

Bybee, J. L. (1995). Diachronic and typological properties of morphology and their implications for representation. Morphological aspects of language processing, 225246.

Canini, K. R., Griffiths, T. L., Vanpaemel, W., \& Kalish, M. L. (2014). Revealing human inductive biases for category learning by simulating cultural transmission. Psychonomic Bulletin \& Review, 21(3), 785-793. 
Chater, N., Clark, A., Goldsmith, J. A., \& Perfors, A. (2015). Empiricism and language learnability. OUP Oxford.

Chater, N., \& Vitányi, P. (2003). Simplicity: A unifying principle in cognitive science?. Trends in cognitive sciences, 7(1), 19-22.

Chollet, F., Keras, (2015), GitHub repository, https://github.com/keras-team/keras

Corbett, G. G. (2009). Suppletion: Typology, markedness, complexity. On inflection, 25-40.

Cotterell, R., Kirov, C., Hulden, M., \& Eisner, J. (2019). On the complexity and typology of inflectional morphological systems. Transactions of the Association for Computational Linguistics, 7, 327342.

Culbertson, J., \& Kirby, S. (2016). Simplicity and specificity in language: Domain-general biases have domain-specific effects. Frontiers in psychology, 6, 1964.

Culbertson, J., \& Newport, E. L. (2015). Harmonic biases in child learners: In support of language universals. Cognition, 139, 71-82.

Culbertson, J., Gagliardi, A., \& Smith, K. (2017). Competition between phonological and semantic cues in noun class learning. Journal of Memory and Language, 92, 343-358.

Culbertson, J., Smolensky, P., \& Legendre, G. (2012). Learning biases predict a word order universal. Cognition, 122(3), 306-329.

Dale, R., \& Lupyan, G. (2012). Understanding the origins of morphological diversity: The linguistic niche hypothesis. Advances in Complex Systems, 15(03n04), 1150017.

Elman, J. L. (1990). Finding structure in time. Cognitive science, 14(2), 179-211.

Elman, J. L. (1991). Distributed representations, simple recurrent networks, and grammatical structure. Machine learning, 7(2-3), 195-225.

Elsner, M., Sims, A. D., Erdmann, A., Hernandez, A., Jaffe, E., Jin, L., ... \& Oh, B. D. (2019). Modeling morphological learning, typology, and change: What can the neural sequence-to-sequence framework contribute?. Journal of Language Modelling, 7(1), 53-98.

Feldman, J. (2016). The simplicity principle in perception and cognition. Wiley Interdisciplinary Reviews: Cognitive Science, 7(5), 330-340.

Finkel, R. \& Stump, G. (2007). Principal parts and morphological typology. Morphology 17.39-75.

Frigo, L., \& McDonald, J. L. (1998). Properties of phonological markers that affect the acquisition of gender-like subclasses. Journal of Memory and Language, 39(2), 218-245.

Futrell, R., \& Levy, R. P. (2018). Do RNNs learn human-like abstract word order preferences?. arXiv preprint arXiv:1811.01866.

Gerken, L., Wilson, R., Gomez, R. L., \& Nurmsoo, E. (2009). The relation between linguistic analogies and lexical categories. In Analogy in grammar: Form and acquisition. Oxford University Press.

Gulordava, K., Bojanowski, P., Grave, E., Linzen, T., \& Baroni, M. (2018). Colorless green recurrent networks dream hierarchically. arXiv preprint arXiv:1803.11138.

Hochreiter, S., \& Schmidhuber, J. (1997). Long short-term memory. Neural computation, 9(8), 1735 1780.

Hockett, C. F. (1954). Two models of grammatical description. Word, 10(2-3), 210-234. 
Hudson Kam, C. L., \& Newport, E. L. (2005). Regularizing unpredictable variation: The roles of adult and child learners in language formation and change. Language learning and development, 1(2), 151-195.

Hudson Kam, C. L., \& Newport, E. L. (2009). Getting it right by getting it wrong: When learners change languages. Cognitive psychology, 59(1), 30-66.

Jordan, M.L (1986): Attractor dynamics and parallelism in a connectionist sequential machine. In: Proceedings of the eighth annual conference of the cognitive science society. Hillsdale, Earlbaum, $531-546$

Kemp, C., \& Regier, T. (2012). Kinship categories across languages reflect general communicative principles. Science, 336(6084), 1049-1054.

Kirby, S., Tamariz, M., Cornish, H., \& Smith, K. (2015). Compression and communication in the cultural evolution of linguistic structure. Cognition, 141, 87-102.

Linzen, T., Dupoux, E., \& Goldberg, Y. (2016). Assessing the ability of LSTMs to learn syntaxsensitive dependencies. Transactions of the Association for Computational Linguistics, 4, 521535.

Malouf, R. (2017). Abstractive morphological learning with a recurrent neural network. Morphology, 27(4), 431-458.

Marzi, C., Ferro, M., Nahli, O., Belik, P., Bompolas, S., \& Pirrelli, V. (2018). Evaluating Inflectional Complexity Crosslinguistically: a Processing Perspective. In Proceedings of the Eleventh International Conference on Language Resources and Evaluation (LREC-2018).

Meinhardt, E., Malouf, R., \& Ackerman, F. Morphology gets more and more complex, unless it doesn'tFinal revision.

Milin, P., Keuleers, E., \& Đurđević, D. (2011). Allomorphic responses in Serbian pseudo-nouns as a result of analogical learning. Acta Linguistica Hungarica, 58(1), 65-84.

Motamedi, Y., Schouwstra, M., Smith, K., Culbertson, J., \& Kirby, S. (2019). Evolving artificial sign languages in the lab: From improvised gesture to systematic sign. Cognition, 192, 103964.

Pothos, E. M., \& Chater, N. (2002). A simplicity principle in unsupervised human categorization. Cognitive Science, 26(3), 303-343.

Ralli, A. (2002). The role of morphology in gender determination: Evidence from Modern Greek. Linguistics, 40(3; ISSU 379), 519-552.

Reeder, P. A., Newport, E. L., \& Aslin, R. N. (2013). From shared contexts to syntactic categories: The role of distributional information in learning linguistic form-classes. Cognitive psychology, 66(1), $30-54$.

Regier, T., Kemp, C., \& Kay, P. (2015). 11 Word Meanings across Languages Support Efficient Communication. The handbook of language emergence, 87, 237.

Saffran, J. R., \& Thiessen, E. D. (2003). Pattern induction by infant language learners. Developmental psychology, 39(3), 484.

Sagot, B. (2013). Comparing complexity measures.

Sapir, E. (1921). Language An introduction to the study of speech. New York: Harcourt, Brace. 
Seyfarth, S., Ackerman, F., \& Malouf, R. (2014). Implicative organization facilitates morphological learning. Proceedings of the 40th Annual Meeting of the Berkeley Linguistics Society.

Shannon, Claude E. (1948). The mathematical theory of communication 1948. The Bell System Technical Journal, 27.379 423, 623-656.

Silvey, C., Kirby, S., \& Smith, K. (2015). Word meanings evolve to selectively preserve distinctions on salient dimensions. Cognitive science, 39(1), 212-226.

Sims, A. D., \& Parker, J. (2016). How inflection class systems work: On the informativity of implicative structure. Word Structure, 9(2), 215-239.

Stump, G. T. (2001). Inflectional morphology: A theory of paradigm structure (Vol. 93). Cambridge University Press.

Tal, S., \& Arnon, I. (2018). SES effects on the use of variation sets in child-directed speech. Journal of child language, 45(6), 1423-1438.

Waterfall, H. R. (2005). A little change is a good thing: Feature theory, language acquisition and variation sets.

Xu, Y., Regier, T., \& Malt, B. C. (2016). Historical semantic chaining and efficient communication: The case of container names. Cognitive science, 40(8), 2081-2094.

Zaslavsky, N., Kemp, C., Tishby, N., \& Regier, T. (2019). Communicative need in colour naming. Cognitive neuropsychology, 1-13.

\section{Appendix}

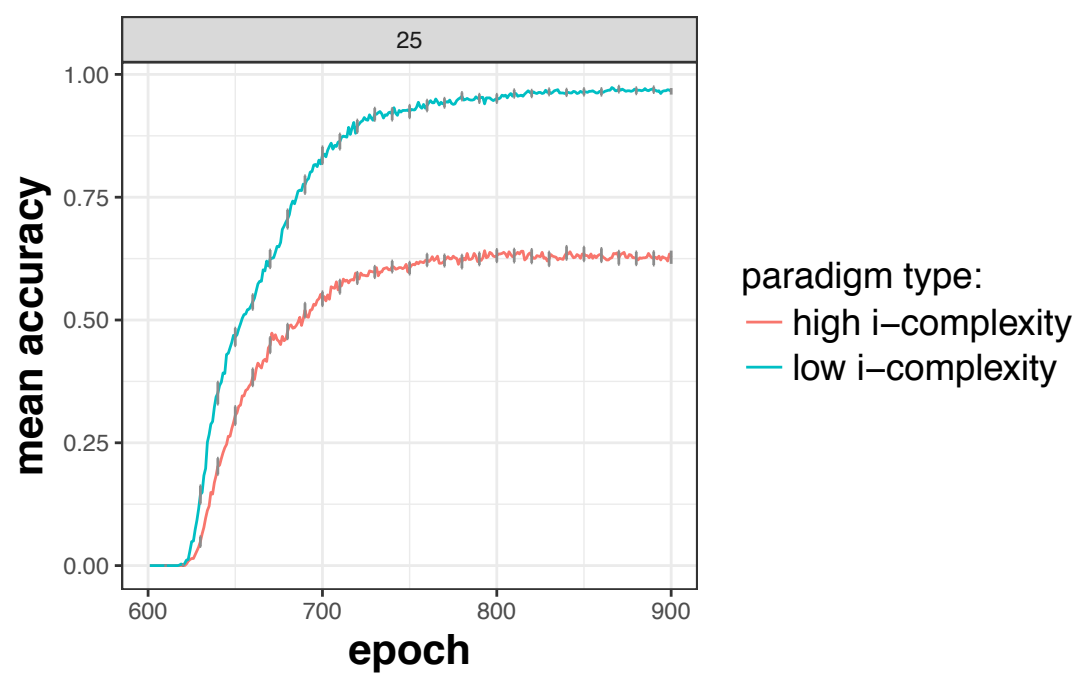

Figure 14. Accuracy of the LSTM networks in generalizing to dual forms of novel stems, after trained on their forms in singular only, for the low i-complexity paradigm (blue) and the high i-complexity paradigm (red). for one network of size 25, with error bars indicating standard error every 10 epochs. Note that the plots start at epoch 600, when the dual forms are introduced to the network (at the beginning of Block 3 ). In all cases accuracy is higher for the low i-complexity paradigm. Networks trained on the high icomplexity paradigm reach accuracy of around $66 \%$ which is the expected chance accuracy when guessing according to the frequent suffix for dual, while networks trained on the low i-complexity paradigm still show accuracy of almost $100 \%$. 